\title{
Progressive Frictional Delamination of an Infinite Elastic Film on a Rigid Substrate Due to In-Plane Point Loading
}

\author{
Marcin Białas
}

Received: 21 June 2010 / Published online: 3 November 2011

(C) The Author(s) 2011. This article is published with open access at Springerlink.com

\begin{abstract}
The paper presents a solution to a delamination problem of an infinite elastic film resting on a rigid substrate and loaded by a monotonically increasing in-plane point force. A rigid-slip contact is assumed between the film and the substrate, leading to the development of two regions at the interface: a damaged zone with a relative slip between the materials, and a region where the interface remains intact. Both film natural and essential boundary conditions are zero on the boundary between these two interfacial zones with the shape of the boundary being a part of the solution. Problem's self-similarity enables us to obtain an approximate distribution of interfacial traction within the delaminated zone and a shape of the zone itself. For film's Poisson's ratio $v=-1$ the approximate solution becomes exact. It is argued that this can be treated as a special case of a rigid film sliding on a rigid substrate. The presented approach can be used to obtain approximate closed-form solutions to similar delamination problems.
\end{abstract}

Keywords Delamination · Friction · Self-similarity $\cdot$ Layered material $\cdot$ Analytic functions Mathematics Subject Classification (2010) 74K35 • 74R99 • 74G10

\section{Introduction}

Growth of interlayer damage leading to failure at the interface is one of the most important problems in mechanics of composite materials, rocks or ceramics. A survey of research in this area can be found, for instance, in the review article by Hutchinson and Suo [12], who discussed mixed mode cracking propagation using the Griffith energy condition. Interfacial failure is specially important for coatings deposited on substrates to improve surface characteristics like, for example, corrosion or wear resistance. If the coating fails, the whole system can be prone to a rapid failure. To improve the mechanical reliability of thin films, their fracture and adhesion properties are of key scientific interest. Many authors analyzed phenomena leading to coating degradation, such as segmentation cracking [10] or film debonding

M. Białas $(\bowtie)$

Institute of Fundamental Technological Research, Pawińskiego 5B, 02-106 Warsaw, Poland

e-mail: mbialas@ippt.gov.pl 
[7], by considering linear elastic systems without taking into account nonlinear effects at the film/substrate interface.

Nonlinear effects at the interface between the coating and the substrate were taken into account by Hue and Evans [11]. They assumed a constant value of shear stress at the interface and performed fracture mechanics analysis of segmentation cracking. In order to measure the ultimate interfacial shear strength Agrawal and Raj [1] assumed given stress distribution at the interface. Thus, without assuming a priori any constitutive relation for this region it was possible to capture elastic deformation, plastic yielding and a softening behaviour associated with film decohesion.

Due to their mathematical simplicity strip models serve as a basis for analytical solutions providing insight into many generally complex problems involving delamination, film cracking or snap-back response, see for example [3, 19]. Even though they provide closed-form solutions they are not able to capture many features intrinsic to two or three dimensional nature of film stiffening or interfacial failure phenomena, like shape of delaminated zone or effect of substrate curvature on failure propagation.

An analytical solution to a $2 \mathrm{D}$ problem of a thin plate adhesively bonded to a surface of an elastic half-space and loaded by a concentrated Mindlin-type force is presented by Selvadurai and Willner [20]. A problem related to a flexure of a plate-like surface layer bonded to an elastic half-space region is discussed by Selvadurai et al. [21]. In this paper the flexure of the coating is induced by a nucleus of thermo-elastic strain acting within the half-space region. In both contributions it is assumed, that the film remains fully bonded to the substrate, with no separation allowed.

The paper presents a solution to a 2D delamination problem of an infinite elastic film resting on a rigid substrate and loaded by a monotonically increasing in-plane point force. A rigid-slip contact is assumed between the film and the substrate, allowing for modeling of interfacial failure in the sliding mode. The presented approach can be used to obtain approximate closed-form solutions of similar delamination phenomena. The problem can be encountered in many practical applications, for example fiber reinforced polymer sheets used in structural strengthening techniques [6] or in laminated glass, where two or more glass plies are bonded together by a polymeric interlayer through treatment at high temperature and pressure in autoclave $[13,17]$.

\section{Problem Formulation}

Let us consider an infinite elastic film of thickness $h$ resting on an infinite rigid foundation. The film is subjected to a monotonically increasing point load $\boldsymbol{P}$ acting in the plane $x y$ on its upper surface, as presented in Fig. 1(a). By assuming rigid-slip interaction between the film and the substrate we have two zones developed at the interface, namely $\Omega$, where the film is displaced due to the external loading $\boldsymbol{P}$, and $\Sigma$ where the structure remains fully bonded with vanishing film displacements. Let $\partial \Omega$ denote the delamination front between the regions $\Omega$ and $\Sigma$, as schematically presented in Fig. 1(b). Assuming the film thickness $h$ to be small as compared to $2 l$, being the characteristic length of the delaminated zone $\Omega$, the problem can be treated as two dimensional with the film in plane stress conditions. The interaction between the film and the substrate is reduced to shear traction $\boldsymbol{\tau}^{\mathrm{f}}$ treated as in-plane body forces acting on the film. Magnitude of traction $\boldsymbol{\tau}^{\mathrm{f}}$ reaches the critical value $\left|\boldsymbol{\tau}^{\mathrm{f}}\right|=\tau^{\mathrm{f}}=$ const within the delaminated zone $\Omega$. The equilibrium equations are

$$
\sigma_{i j, j}+\frac{\tau_{i}^{\mathrm{f}}}{h}+\frac{P_{i}}{h} \delta_{0}=0, \quad(i, j)=1,2,1 \sim x, 2 \sim y,
$$


where $\delta_{0}$ is Dirac distribution. The term $\left(P_{i} / h\right) \delta_{0}$ introduces the effect of concentrated force acting at point $O$. By assuming that the directions of axis $x$ and vector $\boldsymbol{P}$ coincide we have $P_{1}=P_{x}=P, P_{2}=P_{y}=0$. The components of shear traction at the damaged interface have the form

$$
\tau_{1}^{\mathrm{f}}=\tau_{x}^{\mathrm{f}}=\tau^{\mathrm{f}} \cos \theta, \quad \tau_{2}^{\mathrm{f}}=\tau_{y}^{\mathrm{f}}=\tau^{\mathrm{f}} \sin \theta,
$$

where $\theta$ is the angle between the vector of interfacial traction and axis $x$, see Fig. 1(b).

The stress-strain relations for an isotropic elastic medium in plane stress conditions are

$$
\begin{aligned}
\varepsilon_{i i} & =\frac{\sigma_{i i}-v \sigma_{j j}}{E}, \quad(i, j)=x, y, \\
\varepsilon_{x y} & =\frac{1+v}{E} \sigma_{x y}, \\
\varepsilon_{z z} & =-\frac{v}{E}\left(\sigma_{x x}+\sigma_{y y}\right) .
\end{aligned}
$$

The strains are defined as

$$
\varepsilon_{i j}=\frac{1}{2}\left(u_{i, j}+u_{j, i}\right), \quad \varepsilon_{z z}=u_{z, z}, \quad(i, j)=x, y,
$$

where $\boldsymbol{u}$ is the film displacement field.

We assume rigid-slip interface to model the interaction between the film and the foundation. The slip condition is defined in terms of function $F$

$$
F\left(\tau_{x}^{\mathrm{f}}, \tau_{y}^{\mathrm{f}}\right)=\sqrt{\left(\tau_{x}^{\mathrm{f}}\right)^{2}+\left(\tau_{y}^{\mathrm{f}}\right)^{2}}-\tau^{\mathrm{f}}
$$

and we have $F=0$ for $|\dot{\boldsymbol{u}}|>0$ (zone $\Omega$ in Fig. 1(b)) and $F \leq 0$ for $|\dot{\boldsymbol{u}}|=0$ (zone $\Sigma$ in Fig. 1(b)). The constitutive law for the slip at the interface takes the form

$$
\begin{aligned}
& \dot{u}_{i}=-\dot{\lambda} \frac{\partial F}{\partial \tau_{i}^{\mathrm{f}}}, \quad \dot{\lambda} \geq 0, i=(x, y), \\
& F \leq 0, \quad \dot{\lambda} F=0 .
\end{aligned}
$$

By means of (5) and (6) we can write formulae for the sine and cosine of angle $\theta$ between the vector of interfacial traction $\boldsymbol{\tau}^{\mathrm{f}}$ within $\Omega$ and axis $x$

$$
\sin \theta=-\frac{\dot{u}_{y}}{\sqrt{\dot{u}_{x}^{2}+\dot{u}_{y}^{2}}}, \quad \cos \theta=-\frac{\dot{u}_{x}}{\sqrt{\dot{u}_{x}^{2}+\dot{u}_{y}^{2}}} .
$$

Thus, vectors $\boldsymbol{\tau}^{\mathrm{f}}$ and $\dot{\boldsymbol{u}}$ are co-linear and point in opposite directions.

To fully formulate the problem we have to add boundary conditions to (1)-(7). At the delamination front $\partial \Omega$ separating the damaged and intact zones we require

$$
\boldsymbol{u}=\mathbf{0}, \quad \boldsymbol{t}=\boldsymbol{\sigma} . \boldsymbol{n}=\mathbf{0} \quad \text { on } \partial \Omega,
$$

where $\boldsymbol{n}$ is a unit versor normal to the moving boundary $\partial \Omega$ and $\boldsymbol{t}$ is a resultant force acting on $\partial \Omega$. Thus, we have values of both natural and essential boundary conditions prescribed on $\partial \Omega$. The unknown to the problem remains $\partial \Omega$ itself and, as a part of the solution, has to be determined. Our problem belongs to the class of moving boundary problems.

When formulating the problem we need to properly describe the existence of two regions within the film, namely with zero and with non-zero displacement fields. Boundary conditions (8) describe the interaction between them. While within $\Sigma$ we have $\boldsymbol{u}=\mathbf{0}$ by definition, equality $\boldsymbol{u}=\mathbf{0}$ holds also on $\partial \Omega$ due to continuity of displacement field. The 
Fig. 1 (Color online) (a) Infinite elastic film (red) resting on a rigid substrate (grey). In-plane point loading. (b) Two dimensional model: plane stress conditions with interfacial traction $\boldsymbol{\tau}^{\mathrm{f}}$ treated as in-plane body forces (a)

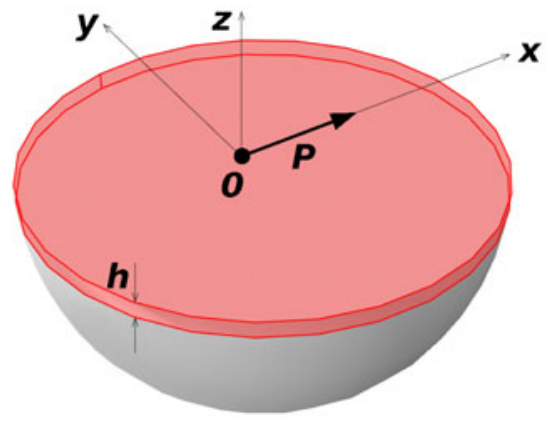

(b)

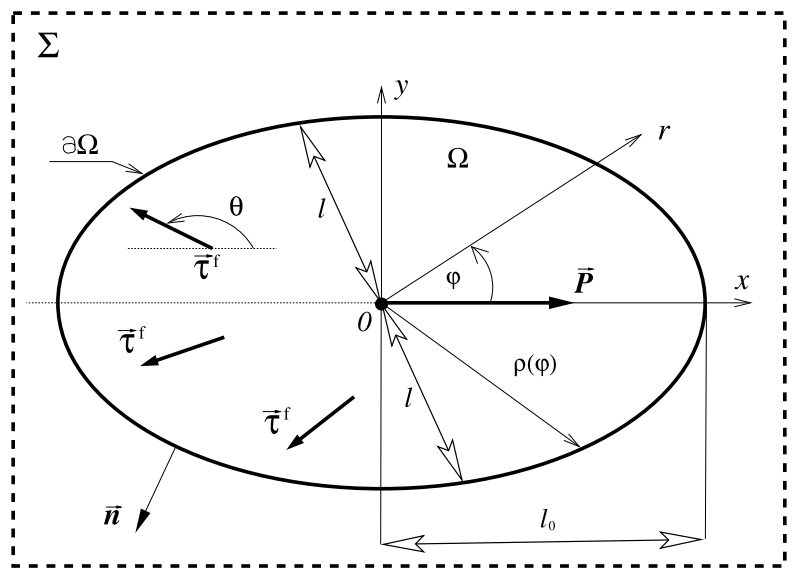

condition $\boldsymbol{t}=\boldsymbol{\sigma} . \boldsymbol{n}=\mathbf{0}$ on $\partial \Omega$ is not so straightforward, however. Vanishing $\boldsymbol{u}$ within $\Sigma$ obviously results in vanishing $\sigma$ in the bonded portion of the film, but it does not necessarily mean that the stress can be continued analytically on the debonded portion passing through the boundary $\partial \Omega$. For example, Gastaldi and Kinderlehrer [8] consider an elastic beam on a rigid table. The precise variational characterization of their problem is not at all trivial and prescribes the formation of a concentrated force at the point between the region where the beam is detached and the region where it is in contact with the substrate. In this context, a rigorous consideration of the character of stress field on $\partial \Omega$ in our case, would probably require advanced variational arguments, and as such it would go beyond the scope of the present paper aimed only at finding the solution to the problem. Keeping all that in mind, one should treat $\boldsymbol{t}=\boldsymbol{\sigma} . \boldsymbol{n}=\mathbf{0}$ as an assumption regarding the interaction between the displaced and bonded portion of the film. The following physical argument can be considered in favor of this assumption. One may infer, albeit tentatively, that any stress concentration might provoke a singularity in the interfacial shear stress, that would certainly overcome the strength of the adhesive bonding, thus producing delamination from the substrate. From this point of view, condition $\boldsymbol{t}=\boldsymbol{\sigma} . \boldsymbol{n}=\mathbf{0}$ on $\partial \Omega$ becomes the only one that is compatible with the limited strength of adhesives introduced by (5) and (6). Capozucca [5], Grande et al. [9] 
and Yuan et al. [24] presented experimentally obtained distribution of strain in fiber reinforced polymer (FRP) sheets bonded to concrete or clay prisms. During pull-push shear tests the sheets were subjected to a loading condition similar to that discussed in the present paper. The decohesion between the FRP reinforcement and the underlaying prisms was the predominant failure mode. The strain gauges located on polymer sheets revealed gradual development of a slip zone between the FRP and the substrate. Recorded strain decreased to zero outside and on the boundary of the detached area. These experimental results provide some support to the condition $\boldsymbol{t}=\boldsymbol{\sigma} . \boldsymbol{n}=\mathbf{0}$ on $\partial \Omega$ assumed in the present analysis.

In the above it is assumed that in the detached area the plate is in slip condition. In general, however, one can think of a more complex situation in which there are some nonslip zones within the detached region. It seems that from physical point of view there are no reasons to exclude a priori such a situation. In this context, the solution sought in the following should be understood as belonging to only one family of possible responses. The fact that it might be the only reasonable response comes from engineering intuition backed up with results of finite element method, which can be obtained totally independently from the forthcoming considerations. The finite element results will be presented in Sect. 8.4.

\section{Dimensional Analysis}

The governing parameters which characterize the considered problem are: $E, \tau^{\mathrm{f}}, v, h, r$, $\varphi$ and $l$. As indicated in Fig. 1(b) parameters $r$ and $\varphi$ are the polar coordinates and $2 l$ is a length of the delaminated zone measured along an arbitrary but constant angle. Instead of $l$ a value of the loading force $P$ could also be used, since both these parameters describe uniquely the delamination process. Both $P$ and $l$ can be prescribed the meaning of time, since only monotonically increasing loading is considered in the paper.

By assuming $l$ and $\tau^{\mathrm{f}}$ to introduce the independent dimensions we can write

$$
\begin{aligned}
\frac{\rho}{l} & =f^{\rho}\left(\varphi, \frac{E}{\tau^{\mathrm{f}}}, v\right), \\
\theta & =f^{\theta}\left(\frac{r}{l}, \varphi, \frac{E}{\tau^{\mathrm{f}}}, v\right),
\end{aligned}
$$

where $\rho$ is a function describing the shape of the boundary $\partial \Omega$ between the intact and damaged interfacial zones. In our analysis the film is assumed to remain elastic during the slip process and to be in plane stress conditions due to its small thickness when compared with characteristic length of damaged zone. For fixed loads $\boldsymbol{\tau}^{\mathrm{f}}$ and $\boldsymbol{P}$ smeared uniformly over the film thickness as done in the equilibrium equation (1), we have the strains, stresses and displacements inversely proportional to $h$ and can write

$$
\begin{gathered}
\varepsilon_{i j} \frac{h}{l}=f_{i j}^{\varepsilon}\left(\frac{r}{l}, \varphi, \frac{E}{\tau^{\mathrm{f}}}, v\right), \\
\frac{\sigma_{i j}}{\tau^{\mathrm{f}}} \frac{h}{l}=f_{i j}^{\sigma}\left(\frac{r}{l}, \varphi, \frac{E}{\tau^{\mathrm{f}}}, v\right), \\
\frac{u_{i} h}{l^{2}}=f_{i}^{\mathrm{u}}\left(\frac{r}{l}, \varphi, \frac{E}{\tau^{\mathrm{f}}}, v\right), \\
(i, j)=x, y .
\end{gathered}
$$

Equations (9) and (10) indicate that the shapes of the moving boundary $\partial \Omega$, as well as the spatial distribution of $\theta$ vary with $l$, while remaining geometrically similar. In fact, we 
encounter here a self-similar phenomenon, where any characteristic of the problem can be written as

$$
\frac{\mathfrak{F}(r, \varphi, t)}{\overline{\mathfrak{F}}(t)}=f\left(\frac{r}{\bar{r}(t)}, \varphi\right) .
$$

In other words, there exist time-dependent scales $\overline{\mathfrak{F}}(t)$ and $\bar{r}(t)$ such that measured in these scales the characteristic $\mathfrak{F}$ becomes time-independent, see [2]. Since only monotonically increasing loading is considered and the parameter $l$ has also the meaning of time, we have $t \sim l=\bar{r}(t)$.

Let us derive a general relation between the loading force $P$ and the characteristic length of the delaminated zone $2 l$. Formula

$$
P=-\tau^{\mathrm{f}} \iint_{\Omega} \cos \theta \mathrm{d} \Omega=-\tau^{\mathrm{f}} \int_{0}^{2 \pi} \int_{0}^{\rho} \cos \theta(r, \varphi) r \mathrm{~d} r \mathrm{~d} \varphi
$$

simply states that the external force has to be equilibrated by the tangential traction at the interface. By normalizing the coordinate $r$ by $l$ and by virtue of (9) and (10) we can write

$$
P=-l^{2} \tau^{\mathrm{f}} \int_{0}^{2 \pi} \int_{0}^{\rho / l} \cos \theta\left(\frac{r}{l}, \varphi\right) \frac{r}{l} \frac{\mathrm{d} r}{l} \mathrm{~d} \varphi=l^{2} \tau^{\mathrm{f}} f^{\mathrm{P}}\left(\frac{E}{\tau^{\mathrm{f}}}, \nu\right),
$$

where

$$
f^{\mathrm{P}}\left(\frac{E}{\tau^{\mathrm{f}}}, v\right)=-\int_{0}^{2 \pi} \int_{0}^{\rho / l} \cos \theta\left(\frac{r}{l}, \varphi\right) \frac{r}{l} \frac{\mathrm{d} r}{l} \mathrm{~d} \varphi .
$$

Thus, there is a quadratic functional dependance between the loading force $P$ and the extent of the delaminated zone.

\section{Homogeneity of Strain, Stress and Displacement Fields}

By virtue of (11) it is easy to show that the strain, stress and displacement fields satisfy the following relations

$$
\begin{aligned}
\varepsilon_{i j}(x, y, l) & =l \varepsilon_{i j}\left(\frac{x}{l}, \frac{y}{l}, 1\right), \\
\sigma_{i j}(x, y, l) & =l \sigma_{i j}\left(\frac{x}{l}, \frac{y}{l}, 1\right), \\
u_{i}(x, y, l) & =l^{2} u_{i}\left(\frac{x}{l}, \frac{y}{l}, 1\right), \\
(i, j) & =x, y,
\end{aligned}
$$

where we have changed space parametrization from polar to Cartesian. Formulae (16) state that functions $\varepsilon_{i j}$ and $\sigma_{i j}$ are homogenous of degree one, and functions $u_{i}$ are homogenous of degree two.

As homogenous, functions $u_{x}$ and $u_{y}$ have to satisfy Euler's theorem

$$
\begin{aligned}
\frac{\partial u_{x}}{\partial x} x+\frac{\partial u_{x}}{\partial y} y+\frac{\partial u_{x}}{\partial l} l & =2 u_{x}, \\
\frac{\partial u_{y}}{\partial x} x+\frac{\partial u_{y}}{\partial y} y+\frac{\partial u_{y}}{\partial l} l & =2 u_{y} .
\end{aligned}
$$


By using (4) we can combine formulae (17) and (18)

$$
2 \varepsilon_{x y}+\frac{x}{y} \varepsilon_{x}+\frac{y}{x} \varepsilon_{y}=\frac{1}{y}\left(2 u_{x}-\frac{\partial u_{x}}{\partial l} l\right)+\frac{1}{y}\left(2 u_{y}-\frac{\partial u_{y}}{\partial l} l\right) .
$$

Consequently, by substituting the Hooke's law (3) into (19) we obtain

$$
\begin{gathered}
2(1+v) \sigma_{x y}+\frac{x}{y}\left(\sigma_{x y}-v \sigma_{y y}\right)+\frac{y}{x}\left(\sigma_{y y}-v \sigma_{x x}\right) \\
=\frac{E}{y}\left(2 u_{x}-\frac{\partial u_{x}}{\partial l} l\right)+\frac{E}{x}\left(2 u_{y}-\frac{\partial u_{y}}{\partial l} l\right) .
\end{gathered}
$$

On the boundary $\partial \Omega$ between the delaminated and intact interfacial zones there is

$$
u_{x}=u_{y}=\frac{\partial u_{x}}{\partial l}=\frac{\partial u_{y}}{\partial l}=0
$$

and (20) reduces to

$$
\left.2(1+v) \sigma_{x y}\right|_{\partial \Omega}+\frac{x}{\rho(x)}\left(\left.\sigma_{x y}\right|_{\partial \Omega}-\left.v \sigma_{y y}\right|_{\partial \Omega}\right)+\frac{\rho(x)}{x}\left(\left.\sigma_{y y}\right|_{\partial \Omega}-\left.v \sigma_{x x}\right|_{\partial \Omega}\right)=0,
$$

where $\rho(x)$ is the function of the boundary in the Cartesian space parametrization and symbol $\left.(\cdot)\right|_{\partial \Omega}$ indicates that the given quantity $(\cdot)$ has to be evaluated on $\partial \Omega$.

By using the second of the boundary conditions (8) we can write

$$
\left.\sigma_{x x}\right|_{\partial \Omega}=-\left.\frac{n_{y}}{n_{x}} \sigma_{x y}\right|_{\partial \Omega},\left.\quad \sigma_{y y}\right|_{\partial \Omega}=-\left.\frac{n_{x}}{n_{y}} \sigma_{x y}\right|_{\partial \Omega},
$$

where $\left\{n_{x}, n_{y}\right\}^{T}$ is a versor normal to $\partial \Omega$. Upon substituting (23) into (22) we obtain

$$
\left.\left[2(1+v)+\frac{x}{\rho(x)}\left(v \frac{n_{x}}{n_{y}}-\frac{n_{y}}{n_{x}}\right)+\frac{\rho(x)}{x}\left(v \frac{n_{y}}{n_{x}}-\frac{n_{x}}{n_{y}}\right)\right] \sigma_{x y}\right|_{\partial \Omega}=0 .
$$

There is $n_{x} / n_{y}=-\rho^{\prime}(x)$ and we can say that either the solution to the differential equation

$$
2(1+v)+\frac{x}{y(x)}\left[\frac{1}{y^{\prime}(x)}-v y^{\prime}(x)\right]+\frac{y(x)}{x}\left[y^{\prime}(x)-\frac{v}{y^{\prime}(x)}\right]=0
$$

provides the unknown boundary $\partial \Omega$, or the stress $\sigma_{x y}$ has to be zero on $\partial \Omega$.

The solution to the differential equation (25) in the polar parametrization of space is given by two functions

$$
r_{1}(\varphi)=C_{1} e^{\sqrt{\nu} \varphi}, \quad r_{2}(\varphi)=C_{2} e^{-\sqrt{\nu} \varphi},
$$

where $C_{1}$ and $C_{2}$ are integration constants. Plots of solutions to (25) are presented in Fig. 2. It is seen that functions $r_{1}(\varphi)$ and $r_{2}(\varphi)$ cannot describe the sought boundary $\partial \Omega$ since, in general, they do not enclose any closed subset of space that could be regarded as the delaminated zone $\Omega$. In other words $r_{i}(0) \neq r_{i}(2 \pi)$ for $v>0, i=1,2$.

The immediate conclusion follows: on the boundary $\partial \Omega$ we have $\sigma_{x y}=0$ and, consequently, by virtue of (23) we deduce that the whole tensor $\sigma$ has to vanish on $\partial \Omega$. The homogeneity of the solution implies that we have to modify the boundary conditions (8) to

$$
\boldsymbol{u}=\mathbf{0}, \quad \boldsymbol{\sigma}=\mathbf{0} \quad \text { on } \partial \Omega .
$$


Fig. 2 Plots of functions $r_{1}(\varphi)$ and $r_{2}(\varphi)$-solutions of (25)
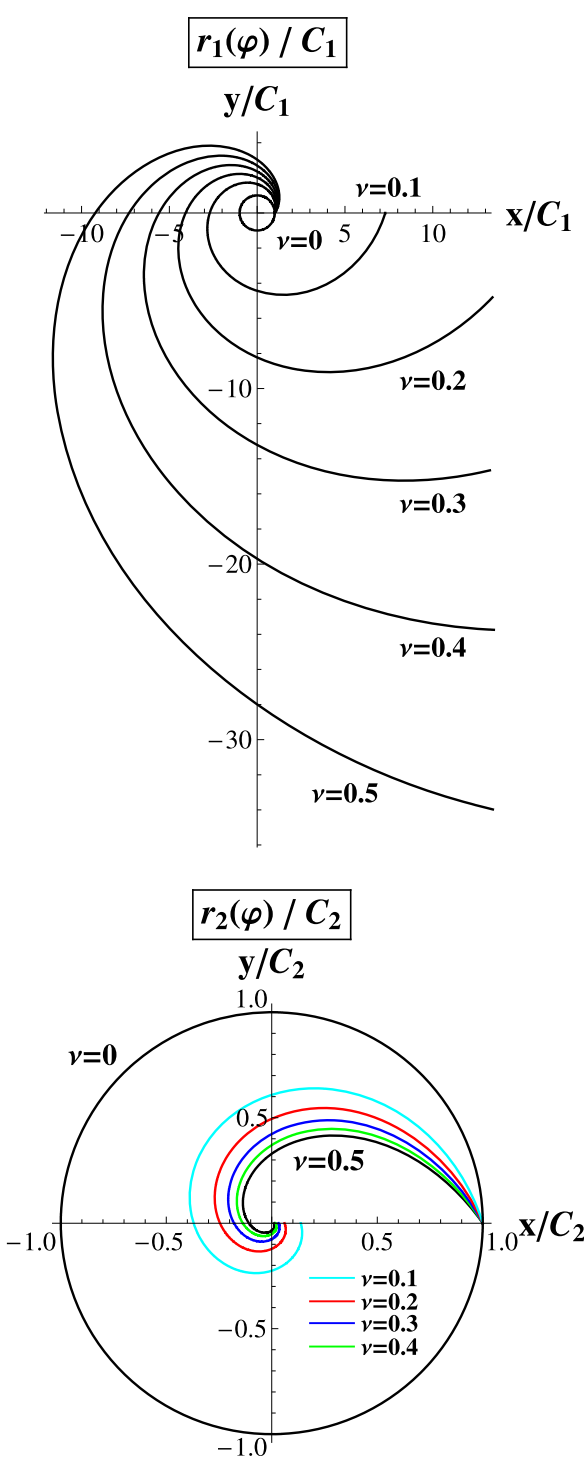

\section{Superposition of Solution}

Due to the fact that the film is assumed to remain elastic during the delamination process, the displacements and the stresses within the film are the superposition of respectively displacements and stresses induced by both the point load $P$ and the forces at the interface. There are zero tractions and displacements at the moving boundary $\partial \Omega$ as imposed by the conditions (27) and we can write

$$
\begin{aligned}
u_{i}(\boldsymbol{x}) & =u_{i}^{\mathrm{P}}(\boldsymbol{x})+u_{i}^{\tau^{\mathrm{f}}}(\boldsymbol{x}), \\
\sigma_{i j}(\boldsymbol{x}) & =\sigma_{i j}^{\mathrm{P}}(\boldsymbol{x})+\sigma_{i j}^{\tau^{\mathrm{f}}}(\boldsymbol{x}),
\end{aligned}
$$

where 


$$
\begin{aligned}
u_{i}^{\mathrm{P}}(\boldsymbol{x}) & =P \tilde{u}_{i}^{x}(\boldsymbol{x} ; \mathbf{0}), \quad \sigma_{i j}^{\mathrm{P}}(\boldsymbol{x})=P \tilde{\sigma}_{i j}^{x}(\boldsymbol{x} ; \mathbf{0}), \\
u_{i}^{\tau^{\mathrm{f}}}(\boldsymbol{x})= & \iint_{\Omega}\left[\tau_{x}^{\mathrm{f}}(\boldsymbol{X}) \tilde{u}_{i}^{x}(\boldsymbol{x} ; \boldsymbol{X})+\tau_{y}^{\mathrm{f}}(\boldsymbol{X}) \tilde{u}_{i}^{y}(\boldsymbol{x} ; \boldsymbol{X})\right] \mathrm{d} \Omega, \\
\sigma_{i j}^{\tau^{\mathrm{f}}}(\boldsymbol{x})= & \iint_{\Omega}\left[\tau_{x}^{\mathrm{f}}(\boldsymbol{X}) \tilde{\sigma}_{i j}^{x}(\boldsymbol{x} ; \boldsymbol{X})+\tau_{y}^{\mathrm{f}}(\boldsymbol{X}) \tilde{\sigma}_{i j}^{y}(\boldsymbol{x} ; \boldsymbol{X})\right] \mathrm{d} \Omega, \\
& (i, j)=x, y .
\end{aligned}
$$

With $j$ being either $x$ or $y$ the functions $\tilde{\boldsymbol{u}}^{j}(\boldsymbol{x} ; \boldsymbol{X})$ and $\tilde{\boldsymbol{\sigma}}^{j}(\boldsymbol{x} ; \boldsymbol{X})$ in (30) are respectively plane stress displacements and stresses at point $\boldsymbol{x}$ resulting from a unit force imposed at point $\boldsymbol{X}$ of an infinite plate of given thickness $h$ and acting in the $j$ direction. In this context, (28) and (29) can be treated as particular forms of Somigliana's identities respectively for displacements and stresses, specified for a structure in plane stress conditions and loaded only by mass forces and a point load, with neither traction nor displacements on the boundary $\partial \Omega$. The Green's functions $\tilde{\boldsymbol{u}}^{j}(\boldsymbol{x} ; \boldsymbol{X})$ and $\tilde{\boldsymbol{\sigma}}^{j}(\boldsymbol{x} ; \boldsymbol{X})$ are derived in Appendix: (65), (66) and (70), (71) are respectively stress and displacement Green's function in the Cartesian parametrization of space, (68), (69) and (72) provide Green's functions written in the polar space parametrization.

Because of the particular choice of Green's functions $\tilde{\boldsymbol{u}}^{j}(\boldsymbol{x} ; \boldsymbol{X})$ and $\tilde{\boldsymbol{\sigma}}^{j}(\boldsymbol{x} ; \boldsymbol{X})$ valid for an infinite plate, we consider an infinite film while searching for the solution. For a given point force $\boldsymbol{P}$ loading the infinite medium we try to find a zone $\Omega$ around $\boldsymbol{P}$ and such a distribution of stresses $\boldsymbol{\tau}^{\mathrm{f}}$ within $\Omega$ that, by virtue of superposition, there is no interaction between $\Omega$ and the surrounding area $\Sigma$, see Fig. 1(b). In order to be so, the condition $\sigma=\mathbf{0}$ on $\partial \Omega$ has to be satisfied. When this is the case, the film within the external region $\Sigma$ is loaded neither by body forces nor on its boundary and, with zero rigid motion, shows no displacements and remains fully bonded to the substrate. Within $\Omega$, on the other hand, the solution to our problem can be obtained by virtue of superposition (28) and (29). Of course, when searching for the distribution of $\boldsymbol{\tau}^{\mathrm{f}}$ within $\Omega$, we should satisfy the slip condition (6), where the meaning of time is prescribed to the monotonically increasing force $\boldsymbol{P}$. Having done so, all equations governing the problem would be satisfied.

In short, to effectively make use of (30) we have to determine two unknowns, namely the distribution of the shear forces at the interface, that is the angle $\theta$, and the shape of the delaminated zone $\Omega$.

Before we proceed, let us analyze the displacement field provided by Green's functions $\tilde{\boldsymbol{u}}^{x}(\boldsymbol{x} ; \mathbf{0})$, that is resulting from a unit force acting on an infinite plate in the $x$ direction and imposed at point $O$. It can be specified from (72) in Appendix for source point coordinates $R=\Phi=0$ in the polar parametrization of space

$$
\begin{aligned}
& \tilde{u}_{x}^{x}(r, \varphi ; \mathbf{0})=\frac{1+v}{4 \pi E h}\left[(3-v) \ln \frac{d}{r}-(1+v) \sin ^{2} \varphi\right], \\
& \tilde{u}_{y}^{x}(r, \varphi ; \mathbf{0})=\frac{(1+v)^{2}}{8 \pi E h} \sin (2 \varphi),
\end{aligned}
$$

where $(r, \varphi)$ are polar coordinates of an observation point. In the above formulas $d$ is an integration constant with a physical meaning of a distance from the loading force to a point on the line co-axial with the loading force vector, where the field $\tilde{\boldsymbol{u}}^{x}(\boldsymbol{x} ; \mathbf{0})$ vanishes. In our case $d$ can in general depend on the loading parameter $l$. A general form of function $d(l)$ can be deduced by taking into account the homogeneity of the displacement field $\boldsymbol{u}$. Remembering that formula (14) states that $P$ is a quadratic function of $l$, we see that in order 
Fig. 3 Plot of plane stress vector field $\tilde{\boldsymbol{u}}^{x}(\boldsymbol{x} ; \mathbf{0})$ resulting from a concentrated unit force acting in the $x$ direction and imposed at point $O$. Dashed line encloses the zone where the horizontal components of the displacement field point in the same direction as the loading force

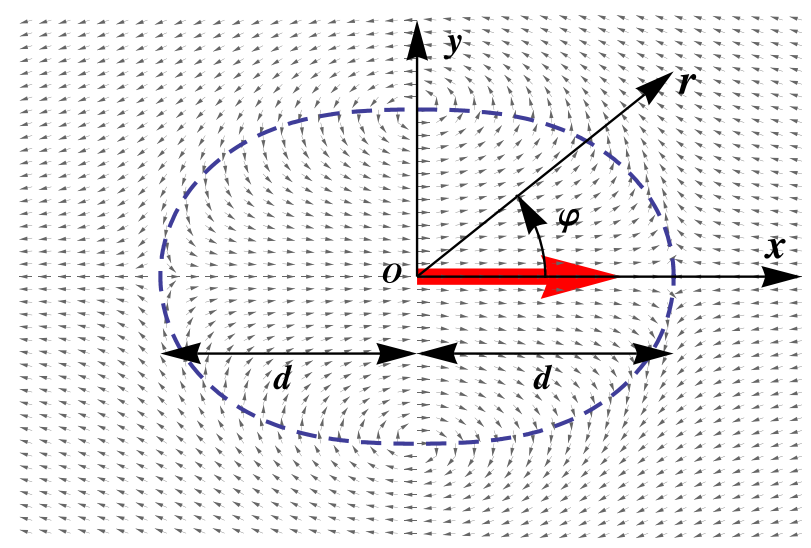

for $u_{x}^{\mathrm{P}}(\boldsymbol{x})$ to be a homogenous function of degree two, $d$ in (31) has to be a homogenous function of $l$ of degree one. As such, it can only be a linear function of $l$ and we can write

$$
d=c l,
$$

where $c$ is as yet an undefined constant.

Vector field $\tilde{\boldsymbol{u}}^{x}(\boldsymbol{x} ; \mathbf{0})$ is presented in Fig. 3. It has a feature typical for two dimensional fundamental problems - there exists a zone around the loading force where the horizontal components of the displacement vectors point in the same direction as the loading force. The extent of this zone is governed by the value of $d$. Outside of this zone the horizontal components of the displacement vectors point in the direction opposite to that of the loading force.

\section{Moving Boundary $\partial \Omega$}

To derive the formula for the moving boundary $\partial \Omega$ let us assume for the moment that we use (29) to calculate the stress field by taking an arbitrary distribution of interfacial traction within the entire space. We also assume the integration area in (29) to be totally arbitrary. In other words, let us calculate stresses in (29) using an arbitrary function $\theta^{*}$ and an arbitrary region $\Omega^{*}$. Without loss of generality we require only the function $\theta^{*}$ and the region $\Omega^{*}$ to be symmetric with respect to axis $x$, and $\Omega^{*}$ to enclose point $O$, where the concentrated force is imposed. With respect to the concentrated force, we want its Cartesian components to be calculated in (29) from formula

$$
P_{x}^{*}=P^{*}=-\tau^{\mathrm{f}} \iint_{\Omega^{*}} \cos \theta^{*} \mathrm{~d} \Omega^{*}, \quad P_{y}^{*}=-\tau^{\mathrm{f}} \iint_{\Omega^{*}} \sin \theta^{*} \mathrm{~d} \Omega^{*}=0,
$$

where the equality $P_{y}^{*}=0$ is guaranteed by the symmetry of function $\theta^{*}$ and region $\Omega^{*}$ with respect to axis $x$. It is seen that $\boldsymbol{P}^{*}$ equilibrates the interfacial forces acting within region $\Omega^{*}$.

We stress out that the choice of function $\theta^{*}$ and integration region $\Omega^{*}$ used for calculation of stresses in (29) and $P_{x}^{*}$ in (33) is totally arbitrary, except for the minor symmetry considerations and the need of $\Omega^{*}$ to include point $O$. In addition to that, $\theta^{*}$ and $\Omega^{*}$ are independent of each other. The situation is presented in Fig. 4, where an arbitrary interfacial traction $\boldsymbol{\tau}^{\mathrm{f}^{*}}$ as well as two arbitrary regions $\Omega_{(1)}^{*}$ and $\Omega_{(2)}^{*}$ have been sketched. They are 
Fig. 4 Integration within arbitrary symmetric regions $\Omega_{(1)}^{*}$ and $\Omega_{(2)}^{*}$ and with an arbitrary vector field $\boldsymbol{\tau}^{\mathrm{f}^{*}}$. Forces $P_{(1)}^{*}$ and $P_{(2)}^{*}$ are both applied at point $O$

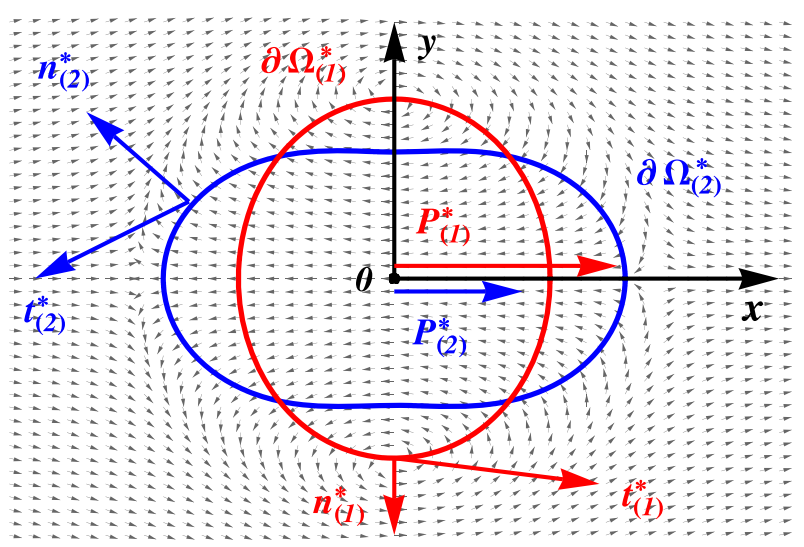

loaded respectively by external forces $\boldsymbol{P}_{(1)}^{*}$ and $\boldsymbol{P}_{(2)}^{*}$, equilibrating the interfacial traction $\boldsymbol{\tau}^{\mathrm{f*}}$ within the integration areas.

In the following all values corresponding to the arbitrary choice of $\Omega^{*}$ and $\theta^{*}$ will have superscript * (for example $\boldsymbol{\sigma}^{*}, \boldsymbol{\tau}^{\mathrm{f}}$ ), in contrast to fields being the solution to our original slip problem (for example $\boldsymbol{\sigma}, \boldsymbol{\tau}^{\mathrm{f}}$ ).

Due to the arbitrary choice of $\Omega^{*}$ and $\theta^{*}$, the stress $\sigma^{*}$ obtained from (29) and resulting from the superposition of external force $\boldsymbol{P}^{*}$ and interfacial traction $\boldsymbol{\tau}^{\mathrm{f}^{*}}$ acting within $\Omega^{*}$ in general does not have to vanish on $\partial \Omega^{*}$. It would vanish, however, when $\boldsymbol{\tau}^{\mathrm{f}^{*}}$ would be the same as the distribution of interfacial stresses at an arbitrary moment of frictional slip in our original delamination problem, and $\partial \Omega^{*}$ would be the corresponding free boundary between detached and undamaged interface.

Since $\boldsymbol{P}^{*}$ equilibrates the interfacial traction within $\Omega^{*}$, forces $\boldsymbol{t}^{*}=\boldsymbol{\sigma}^{*} \cdot \boldsymbol{n}^{*}$ on the boundary $\partial \Omega^{*}$ have no input to the global equilibrium of region $\Omega^{*}$ and, as a result, the following integral has to be zero for any choice of $\theta^{*}$ and $\Omega^{*}$

$$
\int_{\partial \Omega^{*}} \boldsymbol{\sigma}^{*} \cdot \boldsymbol{n}^{*} \mathrm{~d}\left(\partial \Omega^{*}\right)=\mathbf{0},
$$

where $\boldsymbol{n}^{*}$ is a versor normal to $\partial \Omega^{*}$. In the polar parametrization of space we can write equality (34) as

$$
\int_{0}^{2 \pi} \boldsymbol{\sigma}^{*} \cdot \boldsymbol{n}^{*} \mathrm{~d} s^{*}=\mathbf{0}
$$

with integration done along curve $\rho^{*}(\varphi)$.

Let us calculate a variation of equality (35) with respect to function $\rho^{*}$. We obtain

$$
\int_{0}^{2 \pi} \delta \boldsymbol{\sigma}^{*} \cdot \boldsymbol{n}^{*} \mathrm{~d} s^{*}+\int_{0}^{2 \pi} \boldsymbol{\sigma}^{*} \cdot \delta \boldsymbol{n}^{*} \mathrm{~d} s^{*}+\int_{0}^{2 \pi} \boldsymbol{\sigma}^{*} \cdot \boldsymbol{n}^{*} \delta\left(\mathrm{d} s^{*}\right)=\mathbf{0} .
$$

Let us analyze the first term in (36). Writing the multiplication in index notation, using Gauss theorem, commutativity of variation and differentiation and finally equilibrium equation we obtain

$$
\begin{aligned}
\int_{\partial \Omega^{*}} \delta \sigma_{i j}^{*} n_{j} \mathrm{~d}\left(\partial \Omega^{*}\right)= & \iint_{\Omega^{*}}\left(\delta \sigma_{i j}^{*}\right)_{, j} \mathrm{~d} \Omega^{*}=\iint_{\Omega^{*}} \delta\left(\sigma_{i j, j}^{*}\right) \mathrm{d} \Omega^{*} \\
= & -\frac{1}{h} \iint_{\Omega^{*}} \delta\left(\tau_{i}^{\mathrm{f}}+P_{i}^{*} \delta_{0}\right) \mathrm{d} \Omega^{*}=-\frac{1}{h}\left(\delta P_{i}^{*}+\iint_{\Omega^{*}} \delta \tau_{i}^{\mathrm{f}^{*}} \mathrm{~d} \Omega^{*}\right), \\
& (i, j)=1,2,1 \sim x, 2 \sim y
\end{aligned}
$$


It should be noted that $\delta_{0}$ in (37) is Dirac's distribution at point $O$, in contrast to symbol $\delta$ indicating variation.

Let us analyze (36) and (37). The variations in these equations are made with respect to the boundary of integration area $\Omega^{*}$. Because of that values of $\delta \sigma^{*}$ and $\delta P_{x}^{*}=\delta P^{*}$ in general do not vanish, as $\sigma^{*}$ and $P_{x}^{*}=P^{*}$ depend on the choice of $\Omega^{*}$, see (29) and (33) respectively. Variations $\delta \boldsymbol{n}^{*}$ and $\delta\left(\mathrm{d} s^{*}\right)$ also do not vanish, since both $\boldsymbol{n}^{*}$ and $\mathrm{d} s^{*}$ depend on the geometry of $\partial \Omega^{*}$. In the case of $\delta \boldsymbol{\tau}^{\mathrm{f}^{*}}$ the situation is different. As mentioned before, the function $\theta^{*}$ was chosen totally arbitrary and independently from the choice of integration area $\Omega^{*}$. With variation $\delta \tau^{\mathrm{f}^{*}}$ done with respect to $\partial \Omega^{*}$, we can therefore write

$$
\delta \tau_{i}^{\mathrm{f}^{*}}=0
$$

since in the used reasoning $\tau^{\mathrm{f}^{*}}$ does not depend on the choice of $\partial \Omega^{*}$. Equation (36) can then be rewritten as

$$
-\frac{1}{h} \delta \boldsymbol{P}^{*}+\int_{0}^{2 \pi} \boldsymbol{\sigma}^{*} \cdot \delta \boldsymbol{n}^{*} \mathrm{~d} s^{*}+\int_{0}^{2 \pi} \boldsymbol{\sigma}^{*} \cdot \boldsymbol{n}^{*} \delta\left(\mathrm{d} s^{*}\right)=\mathbf{0} .
$$

It is valid for any choice of $\partial \Omega^{*}$ and $\theta^{*}$ and we remind that the variations $\delta \boldsymbol{P}^{*}, \delta \boldsymbol{n}^{*}, \delta\left(\mathrm{d} s^{*}\right)$ are done with respect to the boundary of integration zone $\partial \Omega^{*}$.

Since $\partial \Omega^{*}$ and $\theta^{*}$ in (39) are arbitrary we can make the following choice. Let $\theta^{*}$ be a distribution of interfacial traction for an arbitrary moment of frictional slip in our original delamination problem. In addition to that, let $\partial \Omega^{*}$ be the free boundary between the detached and undamaged interface at the same moment. Then $\boldsymbol{P}^{*}, \theta^{*}$ and $\partial \Omega^{*}$ in (39) become respectively $\boldsymbol{P}, \theta$ and $\partial \Omega$ and describe the distribution $\theta$ of frictional stress within damaged zone $\Omega$ loaded by a point force of magnitude $P$. We recover our original problem and immediately have $\boldsymbol{\sigma}^{*}=\boldsymbol{\sigma}=\mathbf{0}$ on the curve $\rho(\varphi)$. Remembering that $P_{1}=P_{x}=P$ and $P_{2}=P_{y}=0$, equality (39) reduces to

$$
\delta P=0,
$$

which states that the variation of force $\boldsymbol{P}$ made with respect to the boundary of delamination zone has to vanish.

The global equilibrium (33) written in the polar parametrization of space takes the form

$$
P^{*}=-\tau^{\mathrm{f}} \int_{0}^{2 \pi} \int_{0}^{\rho^{*}(\varphi)} \cos \theta^{*}(r, \varphi) r \mathrm{~d} r \mathrm{~d} \varphi .
$$

To make use of result (40) we choose $\rho^{*}(\varphi)$ and $\theta^{*}$ to describe the boundary $\rho(\varphi)$ and angle $\theta$ at an arbitrary moment in our delamination problem. We obtain

$$
\delta P=-\tau^{\mathrm{f}} \delta\left(\int_{0}^{2 \pi} \int_{0}^{\rho(\varphi)} \cos \theta(r, \varphi) r \mathrm{~d} r \mathrm{~d} \varphi\right)=0,
$$

or equivalently

$$
\delta \int_{0}^{2 \pi} I(\rho, \varphi) \mathrm{d} \varphi=0,
$$

where the function

$$
I(\rho, \varphi)=\int_{0}^{\rho(\varphi)} \cos \theta(r, \varphi) r \mathrm{~d} r
$$

has been introduced. Euler's equation for variation (43) reduces now to $\partial I / \partial \rho=0$, providing us with the non-trivial solution

$$
\cos \theta(\rho, \varphi)=0
$$


Thus, on the boundary $\partial \Omega$ between the delaminated and intact interface regions, cosine of angle $\theta$ between vectors of interfacial forces and axis $x$ has to be zero. It allows for identification of boundary $\partial \Omega$, provided the distribution of forces at the interface is known a priori.

\section{Approximate Solution}

Being self-similar, our problem is also self-similar to the delamination resulting from an infinitesimal loading increasing from 0 to $\mathrm{d} P$. Let us therefore discuss the mechanism of slip for this particular situation.

For the displacement field resulting from an infinitesimal loading increasing from 0 to $\mathrm{d} P$, the velocity vectors $\dot{\boldsymbol{u}}$ are co-linear with displacement vectors $\boldsymbol{u}$. For this particular situation we can write

$$
\begin{aligned}
& \sin \theta=\frac{-\dot{u}_{y}}{\sqrt{\left(\dot{u}_{x}\right)^{2}+\left(\dot{u}_{y}\right)^{2}}}=\frac{-u_{y}}{\sqrt{u_{x}^{2}+u_{y}^{2}}}=\frac{-\left(u_{y}^{\mathrm{P}}+u_{y}^{\tau^{\mathrm{f}}}\right)}{\sqrt{\left(u_{x}^{\mathrm{P}}+u_{x}^{\tau^{\mathrm{f}}}\right)^{2}+\left(u_{y}^{\mathrm{P}}+u_{y}^{\tau^{\mathrm{f}}}\right)^{2}}}, \\
& \cos \theta=\frac{-\dot{u}_{x}}{\sqrt{\left(\dot{u}_{x}\right)^{2}+\left(\dot{u}_{y}\right)^{2}}}=\frac{-u_{x}}{\sqrt{u_{x}^{2}+u_{y}^{2}}}=\frac{-\left(u_{x}^{\mathrm{P}}+u_{x}^{\tau^{\mathrm{f}}}\right)}{\sqrt{\left(u_{x}^{\mathrm{P}}+u_{x}^{\tau^{\mathrm{f}}}\right)^{2}+\left(u_{y}^{\mathrm{P}}+u_{y}^{\tau^{\mathrm{f}}}\right)^{2}}},
\end{aligned}
$$

where the superposition (28) has been used.

We introduce the following approximation of $\sin \theta$ and $\cos \theta$, providing us with formulae, which can be easily used in further calculations

$$
\begin{aligned}
& \sin \theta \approx \frac{-u_{y}^{\mathrm{P}}}{\sqrt{\left(u_{x}^{\mathrm{P}}\right)^{2}+\left(u_{y}^{\mathrm{P}}\right)^{2}}}=\frac{-\tilde{u}_{y}^{x}(\boldsymbol{x} ; \mathbf{0})}{\sqrt{\left[\tilde{u}_{x}^{x}(\boldsymbol{x} ; \mathbf{0})\right]^{2}+\left[\tilde{u}_{y}^{x}(\boldsymbol{x} ; \mathbf{0})\right]^{2}}}, \\
& \cos \theta \approx \frac{-u_{x}^{\mathrm{P}}}{\sqrt{\left(u_{x}^{\mathrm{P}}\right)^{2}+\left(u_{y}^{\mathrm{P}}\right)^{2}}}=\frac{-\tilde{u}_{x}^{x}(\boldsymbol{x} ; \mathbf{0})}{\sqrt{\left[\tilde{u}_{x}^{x}(\boldsymbol{x} ; \mathbf{0})\right]^{2}+\left[\tilde{u}_{y}^{x}(\boldsymbol{x} ; \mathbf{0})\right]^{2}}} .
\end{aligned}
$$

Thus, we approximate the exact distribution of interfacial forces for infinitesimal loading $\mathrm{d} P$ by the distribution co-linear with displacement field induced by the concentrated force alone.

Upon substituting (31) into (46) and using $d=c l$, we finally obtain

$$
\begin{aligned}
& \sin \theta \approx \frac{-(1+v) \sin (2 \varphi)}{\sqrt{4\left[(v-3) \ln \frac{r}{c l}-(1+v) \sin ^{2} \varphi\right]^{2}+(1+v)^{2} \sin ^{2}(2 \varphi)}}, \\
& \cos \theta \approx \frac{2\left[(3-v) \ln \frac{r}{c l}+(1+v) \sin ^{2} \varphi\right]}{\sqrt{4\left[(v-3) \ln \frac{r}{c l}-(1+v) \sin ^{2} \varphi\right]^{2}+(1+v)^{2} \sin ^{2}(2 \varphi)}} .
\end{aligned}
$$

Conceptually our deduction was performed for an infinitesimal loading $\mathrm{d} P$ increasing from the undamaged state and, as such, $l$ in (47) should be treated as infinitesimal. However, by virtue of problem's self-similarity, we know that in the normalized coordinates $(r / l, \varphi)$ angle $\theta$ remains constant and this is true for any value of $l>0$. Equations (47) are therefore valid for any positive $l$ and can be used as approximate formulae for cosine and sine of angle $\theta$. 
Fig. 5 Shapes of approximate boundary $\partial \Omega$ between the delaminated and intact interface for $x \geq 0$ and $y \geq 0$ for various values of $v$. Plots of function $\rho(\varphi)$ are symmetric with respect to $x$ and $y$ axis. Zone $\Omega$ is convex for $v \leq 1 / 3$

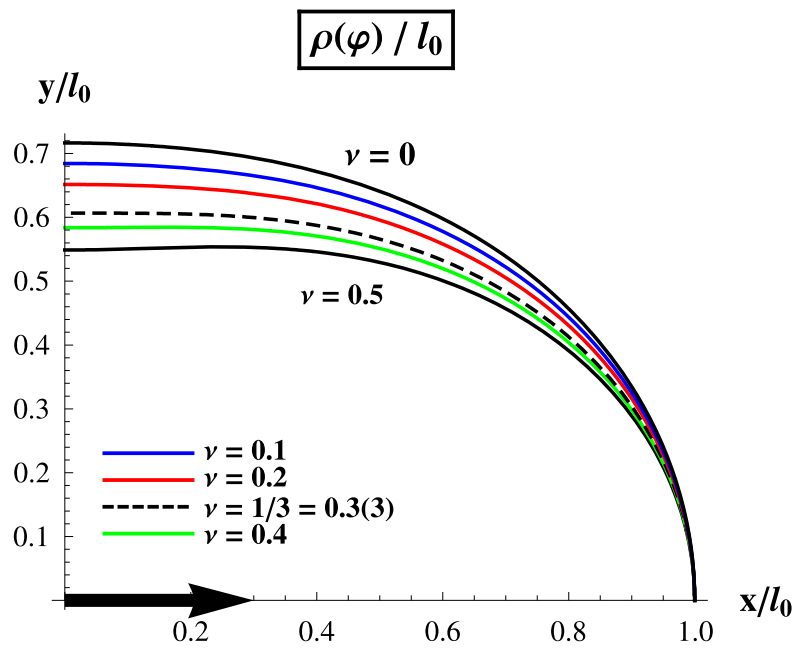

Formula (47) 2 for $\cos \theta$ substituted into condition (44) provides us immediately with an approximate function $\rho(\varphi)$ for the boundary $\partial \Omega$

$$
\rho(\varphi) \approx c l e^{\frac{(1+v) \sin ^{2} \varphi}{\nu-3}} .
$$

Now we can determine the constant $c$ in (47) and in the boundary function (48). For the sake of convenience we choose $l$ to be a distance from the point of external loading $O$ to the boundary $\partial \Omega$, measured along the line coinciding with the vector of point force $\boldsymbol{P}$, as presented in Fig. 1(b). We shall denote this particular $l$ by $l_{0}$. Our choice of parameter $l$ implies that function $\rho(\varphi)$ must equal $l_{0}$ for $\varphi=0$ resulting in $c=1$. The boundary function (48) now takes the form

$$
\rho(\varphi) \approx l_{0} e^{\frac{(1+\nu) \sin ^{2} \varphi}{\nu-3}} .
$$

Plots of function $\rho(\varphi)$ are presented in Fig. 5 for various values of Poisson's ratio $v$, governing the shape of the delaminated zone $\Omega$. For $v>1 / 3$ the delaminated zone is concave, for $v \leq 1 / 3$ it is convex.

In our approximation the forces at the interface are co-linear with displacement vectors resulting from a concentrated force alone. This analogy allows us to explain a physical meaning of condition $\delta P=0$, or equivalently $\cos \theta(\rho, \varphi)=0$, which has enabled us to obtain the moving boundary $\partial \Omega$, by referring to $\tilde{\boldsymbol{u}}^{x}(\boldsymbol{x} ; \mathbf{0})$ vector field. The field $\tilde{\boldsymbol{u}}^{x}(\boldsymbol{x} ; \mathbf{0})$ has this unique feature of a zone around the loading force, where the horizontal components of the displacement vectors point in the same direction as the loading force. Outside of this zone the horizontal components of the displacement vectors point in the direction opposite to that of the concentrated force. On the boundary between these two zones we have $\tilde{u}_{x}^{x}(\boldsymbol{x} ; \mathbf{0})=$ $\cos \theta=0$. Thus, our approximate free boundary $\partial \Omega$ defined by the condition $\cos \theta=0$ is simply the curve dividing the two regions of $\tilde{\boldsymbol{u}}^{x}(\boldsymbol{x} ; \boldsymbol{0})$ field. We also see that the internal zone maximizes value of $P^{*}$ defined by

$$
P^{*}=-\tau^{\mathrm{f}} \iint_{\Omega^{*}} \cos \theta \mathrm{d} \Omega^{*}
$$

and variation $\delta P^{*}$ has to be zero for $\Omega^{*}$ coinciding with this particular region. 
Let us consider consequences of the approximation we made in (46). By taking derivative of (28) we obtain $\mathrm{d} \boldsymbol{u}=\mathrm{d} \boldsymbol{u}^{\mathrm{P}}+\mathrm{d} \boldsymbol{u}^{\tau^{\mathrm{f}}}$, and we see that when calculating $\cos \theta$ and $\sin \theta$ we simply use $\mathrm{d} \boldsymbol{u} \approx \mathrm{d} \boldsymbol{u}^{\mathrm{P}}$. As the concentrated force induces infinite displacements at the loading point, we expect our approximation to render accurate results within a distance not too far from the force. Closer to the moving boundary, we should expect it to generate some error. It manifests itself in the fact that we do not satisfy the condition $\sigma=\mathbf{0}$ exactly at every point of the moving boundary (49). This condition remains satisfied, however, in the weak sense defined by (34), because $\boldsymbol{P}$ equilibrates the interfacial traction within $\Omega$. Accordingly, forces $\boldsymbol{t}=\boldsymbol{\sigma} . \boldsymbol{n}$ on $\partial \Omega$ have no input to the global equilibrium.

\section{Results}

Upon substituting (47) and (49) into formulas (29) and performing the integration we can calculate stresses in the plate within the delaminated area. Due to the complexity of the integrands the integration in (29) can be done only numerically. For $v=-1$ the integrals in (29) simplify and one obtains an analytical solution.

\subsection{Special Case: $v=-1$}

For $v=-1$ we obtain

$$
\sin \theta=0, \quad \cos \theta=-1, \quad \rho(\varphi)=l_{0} .
$$

Thus, the delaminated zone becomes a circle and the components of interfacial forces are given by $\tau_{x}^{\mathrm{f}}=-\tau^{\mathrm{f}}, \tau_{y}^{\mathrm{f}}=0$. The relation between the value of $P$ and the radius of the delaminated zone $l_{0}$ takes the form

$$
P=-\tau^{\mathrm{f}} \int_{0}^{2 \pi} \int_{0}^{\rho} \cos \theta(r, \varphi) r \mathrm{~d} r \mathrm{~d} \varphi=\pi \tau^{\mathrm{f}} l_{0}^{2} .
$$

In the polar parametrization of space formulas (29) for the stresses within the delaminated zone reduce now to

$$
\begin{aligned}
\sigma_{x x} & =-\frac{\tau^{\mathrm{f}} l_{0}^{2} \cos \varphi}{2 h r}+\frac{\tau^{\mathrm{f}}}{2 \pi h} \int_{0}^{2 \pi} \int_{0}^{l_{0}} \frac{(r \cos \varphi-R \cos \Phi) R}{r^{2}+R^{2}-2 r R \cos (\Phi-\varphi)} \mathrm{d} R \mathrm{~d} \Phi \\
& =\frac{\tau^{\mathrm{f}}\left(r^{2}-l_{0}^{2}\right) \cos \varphi}{2 h r}, \\
\sigma_{y y} & =-\sigma_{x x}=\frac{\tau^{\mathrm{f}} l_{0}^{2} \cos \varphi}{2 h r}-\frac{\tau^{\mathrm{f}}}{2 \pi h} \int_{0}^{2 \pi} \int_{0}^{l_{0}} \frac{(r \cos \varphi-R \cos \Phi) R}{r^{2}+R^{2}-2 r R \cos (\Phi-\varphi)} \mathrm{d} R \mathrm{~d} \Phi \\
& =\frac{\tau^{\mathrm{f}}\left(l_{0}^{2}-r^{2}\right) \cos \varphi}{2 h r}, \\
\sigma_{x y} & =-\frac{\tau^{\mathrm{f}} l_{0}^{2} \sin \varphi}{2 h r}+\frac{\tau^{\mathrm{f}}}{2 \pi h} \int_{0}^{2 \pi} \int_{0}^{l_{0}} \frac{(r \sin \varphi-R \sin \Phi) R}{r^{2}+R^{2}-2 r R \cos (\Phi-\varphi)} \mathrm{d} R \mathrm{~d} \Phi \\
& =\frac{\tau^{\mathrm{f}}\left(r^{2}-l_{0}^{2}\right) \sin \varphi}{2 h r},
\end{aligned}
$$

where (51) has been used. Plots of Cartesian components of the stress tensor $\sigma$ are presented in Fig. 6. They satisfy the boundary condition $\boldsymbol{\sigma}=\mathbf{0}$ on $\partial \Omega$. 
Fig. 6 Cartesian components of the stress tensor $\sigma$ within one quadrant of the delaminated zone $\Omega(x \geq 0, y \geq 0)$ obtained for $v=-1$. Arrow indicates the external point force

$$
\frac{\sigma_{\mathrm{xx}}}{\tau^{f}} \frac{h}{l_{0}} ; \quad-\frac{\sigma_{\mathrm{yy}}}{\tau^{f}} \frac{h}{l_{0}}
$$
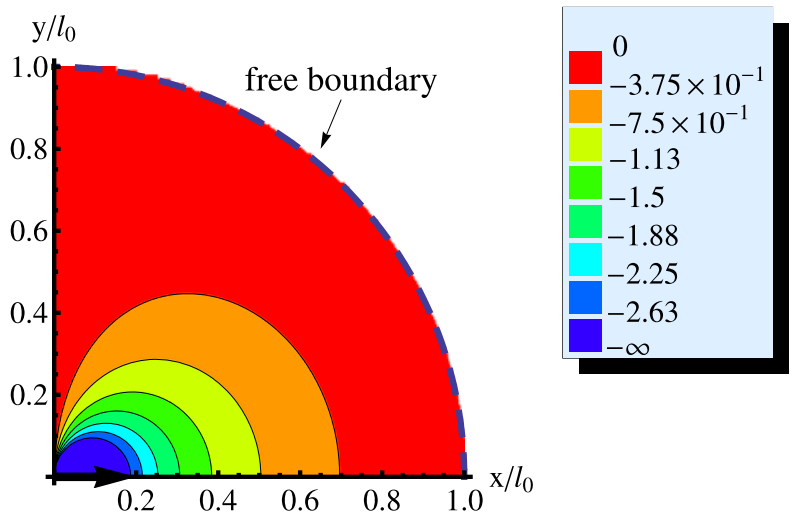

$$
\frac{\sigma_{\mathrm{xy}}}{\tau^{f}} \frac{h}{l_{0}}
$$

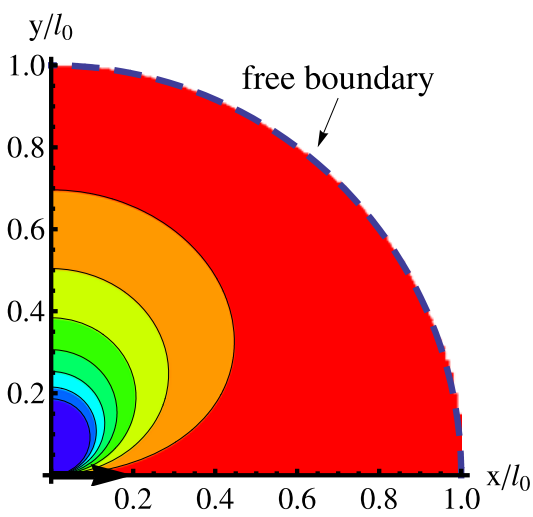

The formulas (28) for the displacement field in our special case provide $\boldsymbol{u}=\mathbf{0}$, which in turn yields vanishing strain tensor $\boldsymbol{\varepsilon}=\mathbf{0}$. In order to explain it, let us notice that the untypical value of Poisson's ratio $v=-1$ results in infinite shear modulus of the plate $G=\frac{E}{2(1+v)}$, thus our material is perfectly rigid for every deformation which is not purely volumetric. In fact, what we obtained is a statically and kinematically admissible solution for a problem of an infinite rigid film resting with frictional contact on a rigid substrate and loaded by an in-plane point force. The approximation (46) we had made for the angle $\theta$ turned out to provide a correct solution for the special case of $v=-1$. Thus, the formulas (50)-(52) constitute an exact solution for our problem specified to a rigid film: they satisfy the boundary conditions (27) and can be accepted in terms of the slip rule (6).

\subsection{Loading Force $P$ Versus Parameter $l_{0}$}

Equation (14) provides a relation between the concentrated force $P$ and the parameter $l$, which in our case has been specified to $l_{0}$. Through (47) and (49) we see that the distribution 
Fig. 7 Plot of function $f^{\mathrm{P}}(v)$

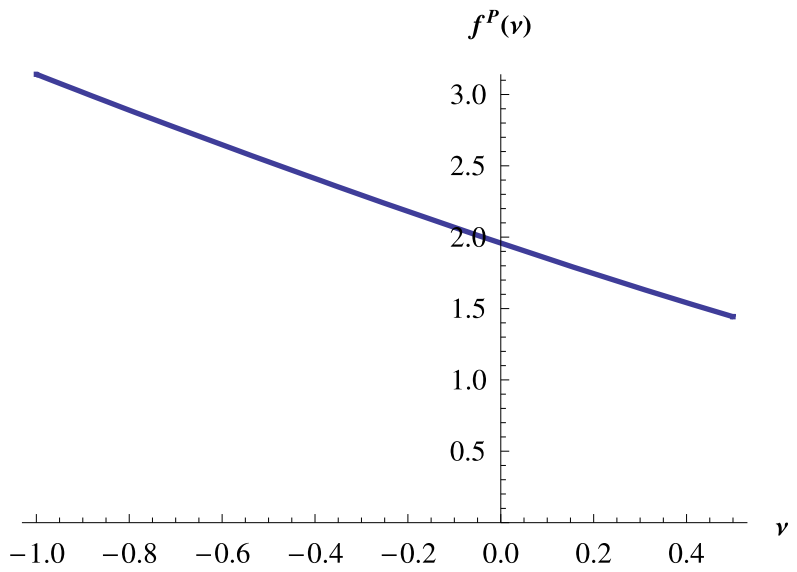

of forces at the interface given by angle $\theta$ and the shape of the delaminated zone $\rho$ do not depend on film's Young's modulus. Thus, $f^{\mathrm{P}}$ appearing in (14) is a function of Poisson's ration $v$ only. Figure 7 presents a plot of function $f^{\mathrm{P}}(v)$ obtained by integration (15) for our specific choice of parameter $l=l_{0}$. In accordance with result (51) it reaches $\pi \approx 3.14$ for $v=-1$.

\subsection{Stress Field}

Figure 8 presents contour stress fields provided by a numerical integration of (29) for Poisson's ratio $v=0.3$. The components $\sigma_{i j}^{\mathrm{P}}$ in (29) introduce the singular behavior of the stress field in the point $O$, where the concentrated force is imposed. As mentioned before, the boundary condition $\boldsymbol{\sigma}=\mathbf{0}$ is not exactly satisfied, which is clearly seen for stress component $\sigma_{y y}$.

\subsection{Comparison with Finite Element Results}

Two dimensional geometry of finite element model is presented in Fig. 9(a). It is a rectangular plate consisting of plane stress elements. The frictional contact between the plate and the rigid substrate is modeled using zero thickness interfacial elements reported by Białas and Mróz [3]. The interface element is presented in Fig. 9(b), with nodes 5, 6, 7, 8 constrained to have zero displacements, as they belong the rigid substrate. Nodes 1, 2, 3, 4 have tangential displacements with components $u_{\xi}$ and $u_{\beta}$. They also define the corresponding plane stress element of the plate.

Fully implicit scheme has been used to integrate the interface constitutive law (6) with slip condition (5). Algorithmic tangent matrices have been calculated for all the elements and penalty stiffness method adopted to enforce the initial rigidity of the interface. Simulations have been performed using Mathematica [16] and finite element package AceFEM [15] developed by Korelc [14]. It allows for an automatic generation of finite element code by simultaneous optimization of expressions within Mathematica [16].

Due to problem symmetry, plate nodes on lines $x=0$ and $y=0$ have been constrained to have zero $u_{y}$ displacements. The concentrated force has been applied at plate node at point $O$ in Fig. 9(a). The plate has been modeled with 40000 rectangular elements, that is with 200 elements in each direction. As long as the loading force is not too big to make the slip zone interact with the plate unconstrained boundaries, the finite element model corresponds 
Fig. 8 Distribution of stresses in the plate within one quadrant of the delaminated zone $\Omega(x \geq 0$, $y \geq 0$ ) obtained for $v=0.3$.

Dashed line indicates the free boundary
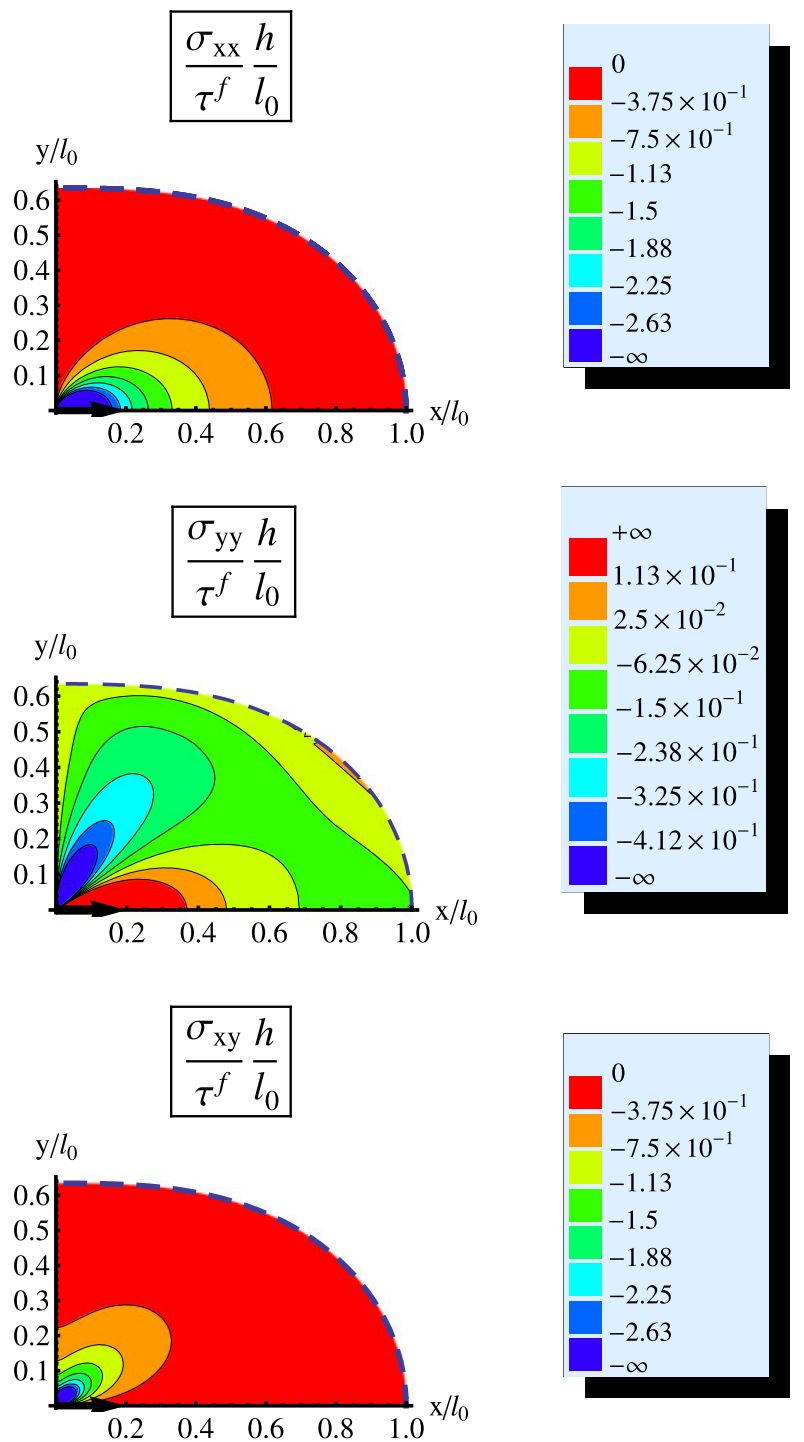

adequately to the original infinite plate problem. During the simulations, the maximum value of the loading force was chosen to result in the characteristic length $l_{0}$ of slip zone to be roughly one fifth of plate width along $x$ axis, as presented in Fig. 9(a). Any numerical difficulties associated with unstable response and plate rigid motion were thus avoided.

Figure 10 presents distribution of stresses within the slip zone obtained from finite element calculations for $v=0.3$. The values have been normalized in order to be easily compared with results presented in Fig. 8. Moreover, the moving boundaries resulting from both the analytical expression (49) and the FEM are indicated. It is seen that the free boundaries agree with each other qualitatively and the complicated shape of FEM boundary is only approximately captured by (49). In particular, the FE slip zone is concave, whereas the curve resulting from function (49) does not enclose a concave area. The $\sigma_{y y}$ stress distribution 
Fig. 9 (a) Geometry of finite element model. (b) Interface element (a)

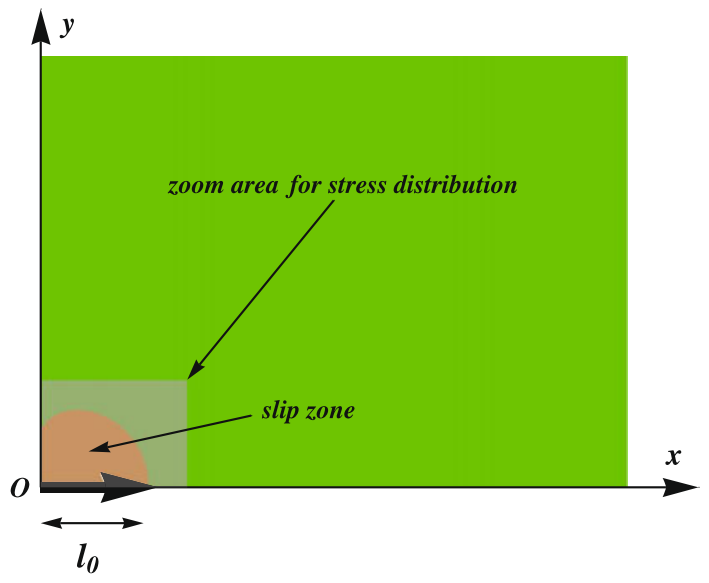

(b)

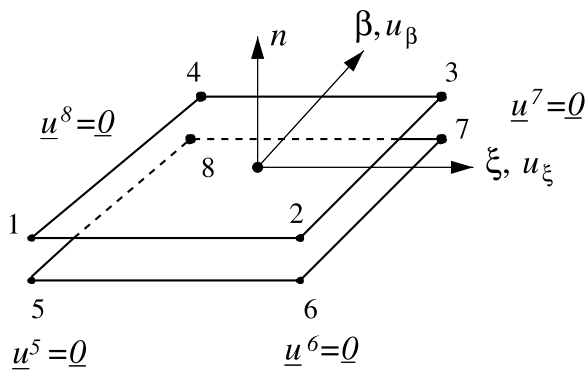

is the most complex and it is seen that the variation of $\sigma_{y y}$ close to the moving boundary in Fig. 8 does not resemble the result obtained from FEM. This is due to the fact that the boundary condition $\sigma_{y y}=0$ on $\partial \Omega$ is not satisfied exactly in Fig. 8. When we move from the free boundary to the location of the loading force however, we see that both FEM and semi-analytical results agree reasonably good with each other and become almost identical. This can bee seen particularly clear in the variation of $\sigma_{x x}$ and $\sigma_{x y}$ stress components, which are rather localized around the loading point. This is in agreement with the previous discussion at the end of Sect. 7.

Figure 11 presents shapes of delaminated zone obtained using finite element method and those provided by the analytical solution (49) for various values of $v$. We see that for all values of Poisson's ratio ranging from 0 to 0.5 the shape of the delaminated zone obtained by finite element method is concave, in contradiction to the analytical result, where it is concave only for $v>1 / 3$. The differences in $\partial \Omega$ provided by the analytical and the numerical approaches tend to be smaller for lower values of $v$. The general trend, however, remains qualitatively preserved and, as expected, shapes of $\partial \Omega$ coincide for $v=-1$.

\section{Summary}

The paper presents an approximate solution to a self-similar problem of frictional delamination. The approximate distribution of interfacial traction enabled us to obtain a closed-form 
Fig. 10 Finite element calculations. Distribution of stresses in the zoom area of Fig. 9(a) for $v=0.3$. The dashed curve indicates the moving boundary given by (49). The continues curve is the moving boundary resulting from FEM

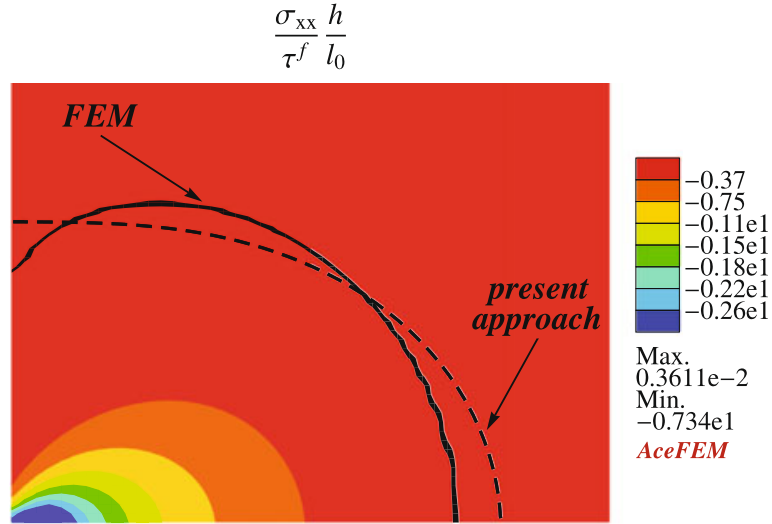

$\frac{\sigma_{\text {yy }}}{\tau^{f}} \frac{h}{l_{0}}$
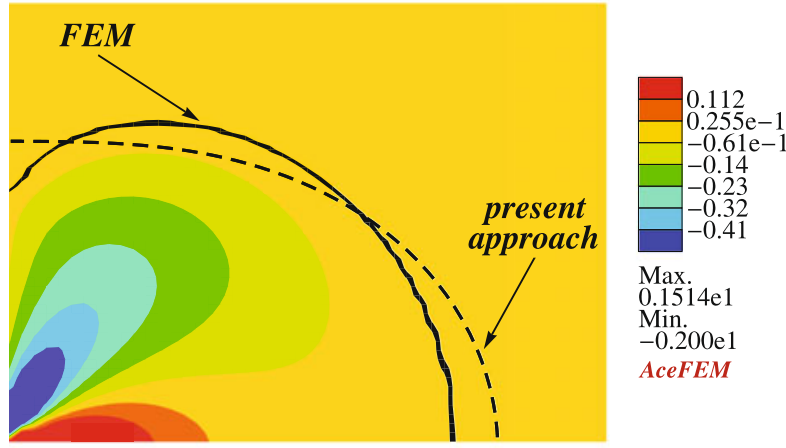

Max.

$0.1514 \mathrm{e} 1$

Min.

$-0.200 \mathrm{e} 1$

AceFEM

$$
\frac{\sigma_{\text {xy }}}{\tau^{f}} \frac{h}{l_{0}}
$$

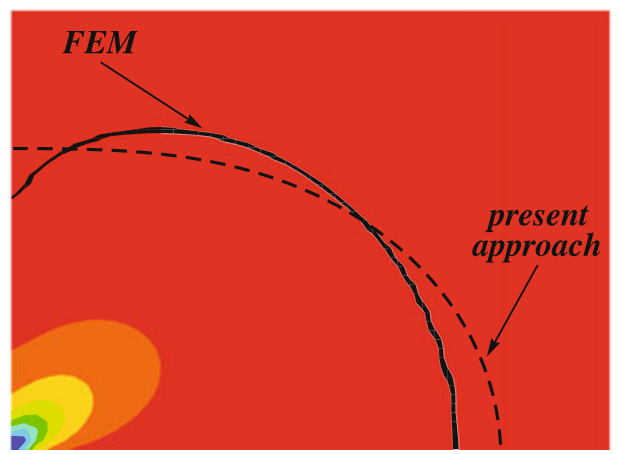

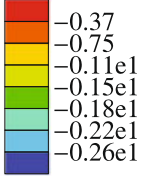

Max.

$0.4232 \mathrm{e}-2$

Min.

$-0.384 \mathrm{e} 1$

AceFEM

solution. The displacement Green's functions of the fundamental 2D problem of an infinite elastic space in plane stress conditions loaded by a point force, provide analytical expressions for the distribution of interfacial traction and the shape of the moving boundary. In context of the approximation, it can be stated that the mechanism of delamination is governed solely by the fundamental solution due to a concentrated force: this solution determines the interfacial stresses and the moving boundary. The delamination area is the same 
Fig. 11 Shapes of delaminated zone obtained using finite element method (green line) and provided by the analytical solution (dashed black line)
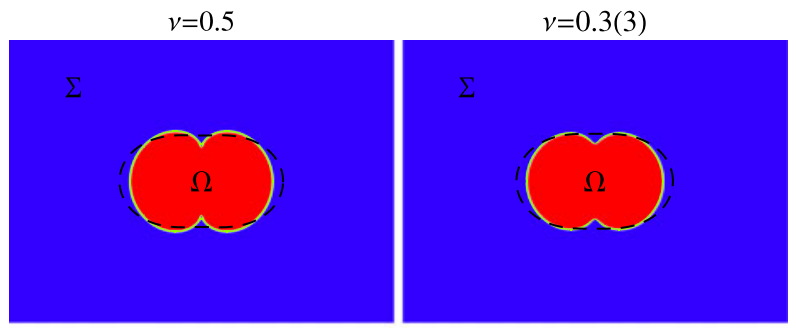

$v=0$

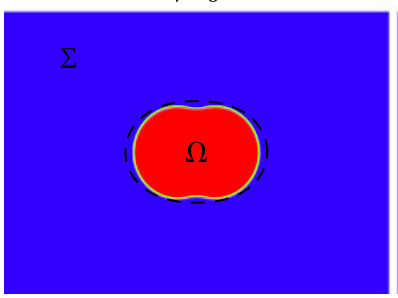

$v=-1$

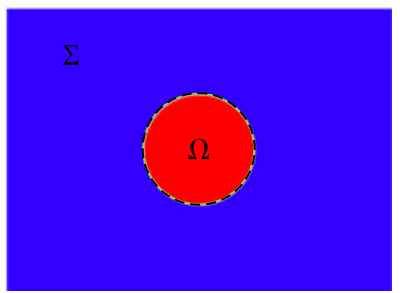

as the region of field $\tilde{\boldsymbol{u}}^{x}(\boldsymbol{x} ; \mathbf{0})$, where the horizontal components of the displacement vectors point in the same direction as the loading force. A special case for $v=-1$ is a solution of rigid film delamination.

The approach presented in the paper can be used to obtain approximate, closed-form solutions for similar delamination problems, for example when considering a film in the shape of a 2D half-space loaded on its edge by an in-plane point force. The algorithm providing an approximate solution is:

1. obtain a solution for the displacement field $\left\{u_{x}^{\mathrm{P}}, u_{y}^{\mathrm{P}}\right\}$ resulting from the point force alone;

2. approximate the distribution of interfacial forces by expressions (46);

3. condition $\delta P=0$ provides a function representing the moving boundary $\partial \Omega$;

4. use the superposition principle to calculate the stress field.

With the value of $\tau^{\mathrm{f}}$ remaining constant during the slip process we make a crude assumption regarding the interface constitutive law. More realistic relation between the shear stress and relative displacement would require the stress first to reach a peak shear strength $\tau^{\text {peak }}$ and then to decrease to a residual value denoted in our case by $\tau^{\mathrm{f}}$, where $\tau^{\mathrm{f}}<\tau^{\text {peak }}$. Such an analysis was performed for example by Palmer and Rice [18], who considered growth of slip surfaces in over-consolidated clay. In the present case, however, the assumption of plane stress conditions is crucial and makes the results applicable to an advanced slip situation only, where the characteristic length $2 l_{0}$ of the damaged zone is big when compared to film thickness $h$. In such a case the value of the interface shear stress is likely to be higher than $\tau^{\mathrm{f}}$ only in the vicinity of the moving boundary $\partial \Omega$ and, with a good approximation, within most of the damaged area $\Omega$ the ultimate shear stress $\tau^{\mathrm{f}}$ is reached. It should also be mentioned that simple interface constitutive laws, as that adopted here, are used for film segmentation cracking problems, rendering solutions which can be successfully used in practice when calculating crack densities, see for example Białas and Mróz [4] or Timm et al. [22].

Acknowledgements The present work was supported by the European Commission through contract No. MERG-CT-2006-036548.

Open Access This article is distributed under the terms of the Creative Commons Attribution Noncommercial License which permits any noncommercial use, distribution, and reproduction in any medium, provided the original author(s) and source are credited. 


\section{Appendix}

The starting point for the derivation of Green's function is the solution for a force at point $O$ of an infinite plate of thickness $h$, as presented in Fig. 12(a). This solution can be found, for example, in Timoshenko and Goodier [23], Article 38. Since only the stresses are given in [23], we will derive here the expressions for the displacement field.

Assuming plane stress conditions, the polar stress components of the problem depicted in Fig. 12(a) have the form, [23]

$$
\sigma=\left[\begin{array}{ll}
\sigma_{r r} & \sigma_{r \varphi} \\
\sigma_{\varphi r} & \sigma_{\varphi \varphi}
\end{array}\right]=P \frac{1-v}{4 \pi h r}\left[\begin{array}{cc}
\frac{3+v}{v-1} \cos \varphi & \sin \varphi \\
\sin \varphi & \cos \varphi
\end{array}\right] .
$$

Plane stress Hook's law provides components of the strain tensor

$$
\begin{aligned}
\epsilon_{r r} & =\frac{\sigma_{r r}-v \sigma_{\varphi \varphi}}{E}=P \frac{\left(v^{2}-2 v-3\right)}{4 \pi E h} \frac{\cos \varphi}{r}, \\
\epsilon_{\varphi \varphi} & =\frac{\sigma_{\varphi \varphi}-v \sigma_{r r}}{E}=P \frac{(1+v)^{2}}{4 \pi E h} \frac{\cos \varphi}{r}, \\
\epsilon_{r \varphi} & =\frac{1+v}{E} \sigma_{r \varphi}=P \frac{\left(1-v^{2}\right)}{4 \pi E h} \frac{\sin \varphi}{r} .
\end{aligned}
$$

Integration of relations

$$
\varepsilon_{r r}=u_{r, r}, \quad \varepsilon_{\varphi \varphi}=\frac{u_{\varphi, \varphi}+u_{r}}{r}, \quad \varepsilon_{r \varphi}=\frac{u_{r, \varphi}+r u_{\varphi, r}-u_{\varphi}}{2 r},
$$

with boundary conditions

$$
\begin{gathered}
u_{\varphi}(r, \varphi=0)=0, \quad u_{\varphi}(r, \varphi=\pi)=0 \quad \text { for } r \geq 0, \\
u_{r}(r=d, \varphi=0)=0,
\end{gathered}
$$

provides the displacement field

$$
\boldsymbol{u}=\left[\begin{array}{l}
u_{r} \\
u_{\varphi}
\end{array}\right]=P \frac{1+v}{4 \pi E h}\left[\begin{array}{c}
\frac{3+2 v-v^{2}}{1+v} \cos \varphi \ln \frac{d}{r} \\
\left(1+v+(3-v) \ln \frac{r}{d}\right) \sin \varphi
\end{array}\right] .
$$

The first two conditions (56) simply enforce the symmetry of the displacement field with respect to the axis co-linear with the external force $P$. The last one means that the plate does not displace at an arbitrary point on the symmetry axis at the distance $d$ from the loading force. There are no physical prerequisites to chose any specific value of $d$ and, as such, it can be treated just like an undefined, superfluous integration constant.

To derive the Green's functions we shall consider two different Cartesian coordinate systems, namely $\left\{x^{I}, y^{I}\right\}$ and $\left\{x^{I I}, y^{I I}\right\}$. In the first one the loading force $P$ has the direction of axis $x^{I}$, in the second one it is aligned with the axis $y^{I I}$ as presented in Fig. 12(b) and Fig. 12(c). The following formulae relate the polar with the two Cartesian coordinate systems

$$
\begin{aligned}
\sin \varphi & =\frac{y^{I}}{\sqrt{\left(x^{I}\right)^{2}+\left(y^{I}\right)^{2}}}=\frac{-x^{I I}}{\sqrt{\left(x^{I I}\right)^{2}+\left(y^{I I}\right)^{2}}}, \\
\cos \varphi & =\frac{x^{I}}{\sqrt{\left(x^{I}\right)^{2}+\left(y^{I}\right)^{2}}}=\frac{y^{I I}}{\sqrt{\left(x^{I I}\right)^{2}+\left(y^{I I}\right)^{2}}}, \\
r & =\sqrt{\left(x^{I}\right)^{2}+\left(y^{I}\right)^{2}}=\sqrt{\left(x^{I I}\right)^{2}+\left(y^{I I}\right)^{2}} .
\end{aligned}
$$


Fig. 12 (a) Infinite film loaded by in-plane point force. (b),

(c) Two Cartesian coordinate systems $\left\{x^{I}, y^{I}\right\}$ and $\left\{x^{I I}, y^{I I}\right\}$ (a)

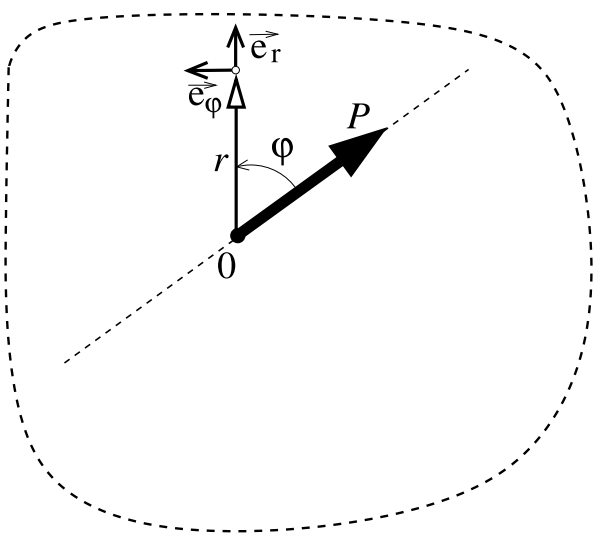

(b)

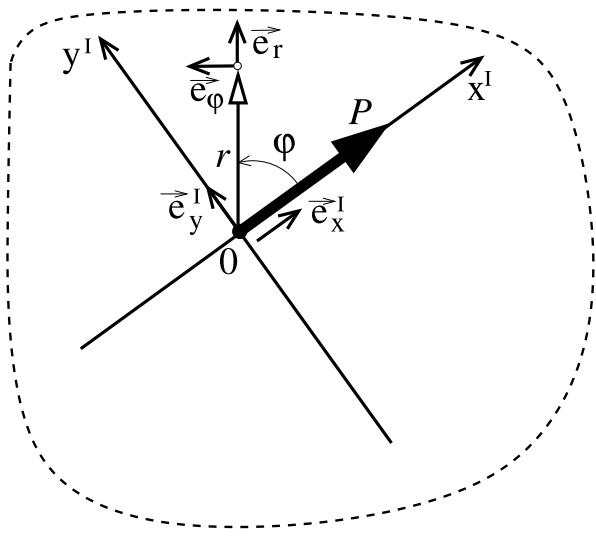

(c)

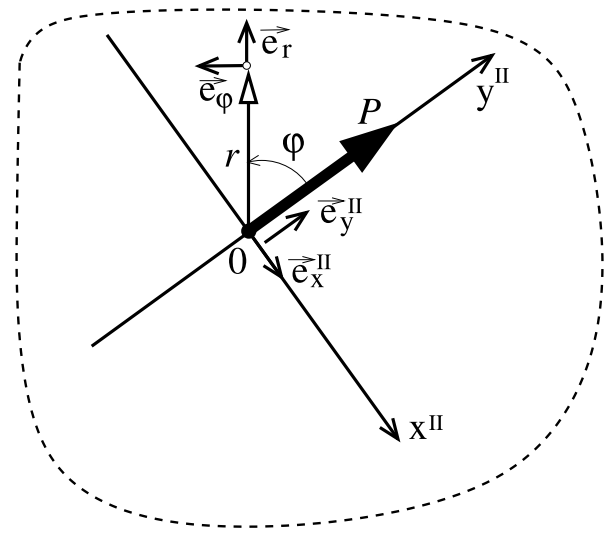


Additionally, the following relations hold

$$
\left[\mathbf{e}_{r}, \mathbf{e}_{\varphi}\right]^{T}=\boldsymbol{B}^{I}\left[\mathbf{e}_{x}^{I}, \mathbf{e}_{y}^{I}\right]^{T}=\boldsymbol{B}^{I I}\left[\mathbf{e}_{x}^{I I}, \mathbf{e}_{y}^{I I}\right]^{T},
$$

where the matrices $\boldsymbol{B}^{I}$ and $\boldsymbol{B}^{I I}$ have the form

$$
\boldsymbol{B}^{I}=\left[\begin{array}{cc}
\cos \varphi & \sin \varphi \\
-\sin \varphi & \cos \varphi
\end{array}\right], \quad \boldsymbol{B}^{I I}=\left[\begin{array}{cc}
-\sin \varphi & \cos \varphi \\
-\cos \varphi & -\sin \varphi
\end{array}\right]
$$

The transformations $\left(\boldsymbol{B}^{I}\right)^{T} \boldsymbol{\sigma} \boldsymbol{B}^{I}$ and $\left(\boldsymbol{B}^{I I}\right)^{T} \boldsymbol{\sigma} \boldsymbol{B}^{I I}$ together with formulas (53), (58) and (60) provide stress components respectively in the first and in the second Cartesian coordinate system. We obtain

$$
\begin{aligned}
& \sigma_{x^{I} x^{I}}=P \frac{x^{I}\left[(v-1)\left(y^{I}\right)^{2}-(3+v)\left(x^{I}\right)^{2}\right]}{4 \pi h\left[\left(x^{I}\right)^{2}+\left(y^{I}\right)^{2}\right]^{2}}, \\
& \sigma_{y^{I} y^{I}}=P \frac{x^{I}\left[(1-v)\left(x^{I}\right)^{2}-(1+3 v)\left(y^{I}\right)^{2}\right]}{4 \pi h\left[\left(x^{I}\right)^{2}+\left(y^{I}\right)^{2}\right]^{2}}, \\
& \sigma_{x^{I} y^{I}}=P \frac{y^{I}\left[(v-1)\left(y^{I}\right)^{2}-(3+v)\left(x^{I}\right)^{2}\right]}{4 \pi h\left[\left(x^{I}\right)^{2}+\left(y^{I}\right)^{2}\right]^{2}}
\end{aligned}
$$

in the first Cartesian coordinate system and

$$
\begin{aligned}
& \sigma_{x^{I I} x^{I I}}=P \frac{y^{I I}\left[(1-v)\left(y^{I I}\right)^{2}-(1+3 v)\left(x^{I I}\right)^{2}\right]}{4 \pi h\left[\left(x^{I I}\right)^{2}+\left(y^{I I}\right)^{2}\right]^{2}}, \\
& \sigma_{y^{I I} y^{I I}}=P \frac{y^{I I}\left[(v-1)\left(x^{I I}\right)^{2}-(3+v)\left(y^{I I}\right)^{2}\right]}{4 \pi h\left[\left(x^{I I}\right)^{2}+\left(y^{I I}\right)^{2}\right]^{2}}, \\
& \sigma_{x^{I I} y^{I I}}=P \frac{x^{I I}\left[(v-1)\left(x^{I I}\right)^{2}-(3+v)\left(y^{I I}\right)^{2}\right]}{4 \pi h\left[\left(x^{I I}\right)^{2}+\left(y^{I I}\right)^{2}\right]^{2}}
\end{aligned}
$$

in the second.

The transformations $\left(\boldsymbol{B}^{I}\right)^{T} \boldsymbol{u}$ and $\left(\boldsymbol{B}^{I I}\right)^{T} \boldsymbol{u}$ together with formulas (57), (58) and (60) provide displacement components respectively in the first and in the second Cartesian coordinate system. We obtain

$$
\begin{aligned}
& u_{x^{I}}=P \frac{1+v}{4 \pi E h}\left[(3-v) \ln \frac{d^{I}}{\sqrt{\left(x^{I}\right)^{2}+\left(y^{I}\right)^{2}}}-(1+v) \frac{\left(y^{I}\right)^{2}}{\left(x^{I}\right)^{2}+\left(y^{I}\right)^{2}}\right], \\
& u_{y^{I}}=P \frac{(1+v)^{2}}{4 \pi E h} \frac{x^{I} y^{I}}{\left(x^{I}\right)^{2}+\left(y^{I}\right)^{2}}
\end{aligned}
$$

in the first Cartesian coordinate system and

$$
\begin{aligned}
& u_{x^{I I}}=P \frac{(1+v)^{2}}{4 \pi E h} \frac{x^{I I} y^{I I}}{\left(x^{I I}\right)^{2}+\left(y^{I I}\right)^{2}} \\
& u_{y^{I I}}=P \frac{1+v}{4 \pi E h}\left[(3-v) \ln \frac{d^{I I}}{\sqrt{\left(x^{I I}\right)^{2}+\left(y^{I I}\right)^{2}}}-(1+v) \frac{\left(x^{I I}\right)^{2}}{\left(x^{I I}\right)^{2}+\left(y^{I I}\right)^{2}}\right]
\end{aligned}
$$

in the second. The auxiliary parameter $d$ in (57) has been changed to $d^{I}$ and $d^{I I}$ respectively in (63) and (64).

Equations (61) and (62) can be used to obtain stress Green's functions in a general Cartesian coordinate system $\{x, y\}$. For a unit force acting in the positive $x$ direction we have 


$$
\begin{aligned}
& \tilde{\sigma}_{x x}^{x}=\frac{(x-X)\left[(v-1)(y-Y)^{2}-(3+v)(x-X)^{2}\right]}{4 \pi h\left[(x-X)^{2}+(y-Y)^{2}\right]^{2}}, \\
& \tilde{\sigma}_{y y}^{x}=\frac{(x-X)\left[(1-v)(x-X)^{2}-(1+3 v)(y-Y)^{2}\right]}{4 \pi h\left[(x-X)^{2}+(y-Y)^{2}\right]^{2}}, \\
& \tilde{\sigma}_{x y}^{x}=\frac{(y-Y)\left[(v-1)(y-Y)^{2}-(3+v)(x-X)^{2}\right]}{4 \pi h\left[(x-X)^{2}+(y-Y)^{2}\right]^{2}} .
\end{aligned}
$$

Unit force aligned with the positive $y$ direction induces the stresses

$$
\begin{aligned}
& \tilde{\sigma}_{x x}^{y}=\frac{(y-Y)\left[(1-v)(y-Y)^{2}-(1+3 v)(x-X)^{2}\right]}{4 \pi h\left[(x-X)^{2}+(y-Y)^{2}\right]^{2}}, \\
& \tilde{\sigma}_{y y}^{y}=\frac{(y-Y)\left[(v-1)(x-X)^{2}-(3+v)(y-Y)^{2}\right]}{4 \pi h\left[(x-X)^{2}+(y-Y)^{2}\right]^{2}}, \\
& \tilde{\sigma}_{x y}^{y}=\frac{(x-X)\left[(v-1)(x-X)^{2}-(3+v)(y-Y)^{2}\right]}{4 \pi h\left[(x-X)^{2}+(y-Y)^{2}\right]^{2}} .
\end{aligned}
$$

In (65) and (66) small letters $(x, y)$ indicate an observation point and capital letters $(X, Y)$ stand for a source point.

Equations (65) and (66) can be re-written in the polar parametrization of space. By substituting

$$
x=r \cos \varphi, \quad y=r \sin \varphi, \quad X=R \cos \Phi, \quad y=R \sin \Phi
$$

into (65) and (66) we obtain

$$
\begin{aligned}
\tilde{\sigma}_{x x}^{x}= & (R \cos \Phi-r \cos \varphi) \\
& \cdot \frac{(3+v)(R \cos \Phi-r \cos \varphi)^{2}+(1-v)(R \sin \Phi-r \sin \varphi)^{2}}{4 \pi h\left[r^{2}+R^{2}-2 r R \cos (\Phi-\varphi)\right]^{2}}, \\
\tilde{\sigma}_{y y}^{x}= & (R \cos \Phi-r \cos \varphi) \\
& \cdot \frac{(v-1)(R \cos \Phi-r \cos \varphi)^{2}+(1+3 v)(R \sin \Phi-r \sin \varphi)^{2}}{4 \pi h\left[r^{2}+R^{2}-2 r R \cos (\Phi-\varphi)\right]^{2}}, \\
\tilde{\sigma}_{x y}^{x}= & (R \sin \Phi-r \sin \varphi) \\
& \cdot \frac{(3+v)(R \cos \Phi-r \cos \varphi)^{2}+(1-v)(R \sin \Phi-r \sin \varphi)^{2}}{4 \pi h\left[r^{2}+R^{2}-2 r R \cos (\Phi-\varphi)\right]^{2}}
\end{aligned}
$$

and respectively

$$
\begin{aligned}
\tilde{\sigma}_{x x}^{y}= & (R \sin \Phi-r \sin \varphi) \\
& \cdot \frac{(1+3 v)(R \cos \Phi-r \cos \varphi)^{2}-(1-v)(R \sin \Phi-r \sin \varphi)^{2}}{4 \pi h\left[r^{2}+R^{2}-2 r R \cos (\Phi-\varphi)\right]^{2}}, \\
\tilde{\sigma}_{y y}^{y}= & (R \sin \Phi-r \sin \varphi) \\
& \cdot \frac{(1-v)(R \cos \Phi-r \cos \varphi)^{2}+(3+v)(R \sin \Phi-r \sin \varphi)^{2}}{4 \pi h\left[r^{2}+R^{2}-2 r R \cos (\Phi-\varphi)\right]^{2}}, \\
\tilde{\sigma}_{x y}^{y}= & (R \cos \Phi-r \cos \varphi) \\
& \cdot \frac{(1-v)(R \cos \Phi-r \cos \varphi)^{2}+(3+v)(R \sin \Phi-r \sin \varphi)^{2}}{4 \pi h\left[r^{2}+R^{2}-2 r R \cos (\Phi-\varphi)\right]^{2}} .
\end{aligned}
$$

Here, again, small letters $(r, \varphi)$ indicate an observation point and capital $(R, \Phi)$ stand for a source point. 
Equations (63) and (64) can be used to obtain displacement Green's functions in a general Cartesian coordinate system $\{x, y\}$. For a unit force acting in a positive $x$ direction we simply have

$$
\begin{aligned}
\tilde{u}_{x}^{x}= & \frac{1+v}{4 \pi E h}\left[(3-v) \ln \frac{d^{x}(X, Y)}{\sqrt{(x-X)^{2}+(y-Y)^{2}}}\right. \\
& \left.-(1+v) \frac{(y-Y)^{2}}{(x-X)^{2}+(y-Y)^{2}}\right], \\
\tilde{u}_{y}^{x}= & \frac{(1+v)^{2}}{4 \pi E h} \frac{(x-X)(y-Y)}{(x-X)^{2}+(y-Y)^{2}} .
\end{aligned}
$$

Unit force aligned with a positive $y$ direction results in the displacements

$$
\begin{aligned}
\tilde{u}_{x}^{y}= & \frac{(1+v)^{2}}{4 \pi E h} \frac{(x-X)(y-Y)}{(x-X)^{2}+(y-Y)^{2}} \\
\tilde{u}_{y}^{y}= & \frac{1+v}{4 \pi E h}\left[(3-v) \ln \frac{d^{y}(X, Y)}{\sqrt{(x-X)^{2}+(y-Y)^{2}}}\right. \\
& \left.-(1+v) \frac{(x-X)^{2}}{(x-X)^{2}+(y-Y)^{2}}\right] .
\end{aligned}
$$

The upper index $j$ in $\tilde{u}_{i}^{j}$ stands for the source direction, the lower index $i$ stands for displacement direction. Again, the small letters $(x, y)$ in (70) and (71) indicate an observation point, the capital $(X, Y)$ a source point. The auxiliary parameters $d^{I}$ and $d^{I I}$ have been changed respectively to $d^{x}$ and $d^{y}$. In general, the Green's functions can depend on a position of source point and this fact has been indicated in (70) and (71) by writing respectively $d^{x}(X, Y)$ and $d^{y}(X, Y)$. There is of course $d=d^{I}=d^{x}(0,0)$ and $d^{I I}=d^{y}(0,0)$.

After substituting (67) into (70) and (71) we obtain displacement Green's functions in the polar parametrization of space

$$
\begin{aligned}
\tilde{u}_{x}^{x}= & \frac{1+v}{4 \pi E h}\left[(3-v) \ln \frac{d^{x}(R, \Phi)}{\sqrt{r^{2}+R^{2}-2 r R \cos (\Phi-\varphi)}}\right. \\
& \left.-(1+v) \frac{(R \sin \Phi-r \sin \varphi)^{2}}{r^{2}+R^{2}-2 r R \cos (\Phi-\varphi)}\right] \\
\tilde{u}_{y}^{x}= & \tilde{u}_{x}^{y}=\frac{(1+v)^{2}}{4 \pi E h} \frac{(R \cos \Phi-r \cos \varphi)(R \sin \Phi-r \sin \varphi)}{r^{2}+R^{2}-2 r R \cos (\Phi-\varphi)}, \\
\tilde{u}_{y}^{y}= & \frac{1+v}{4 \pi E h}\left[(3-v) \ln \frac{d^{y}(R, \Phi)}{\sqrt{r^{2}+R^{2}-2 r R \cos (\Phi-\varphi)}}\right. \\
& \left.-(1+v) \frac{(R \cos \Phi-r \cos \varphi)^{2}}{r^{2}+R^{2}-2 r R \cos (\Phi-\varphi)}\right] .
\end{aligned}
$$

Small letters $(r, \varphi)$ indicate an observation point and capital $(R, \Phi)$ stand for a source point. In consistency with the previous notation, there is $d=d^{I}=d^{x}(0,0)$ and $d^{I I}=d^{y}(0,0)$.

\section{References}

1. Agrawal, D.C., Raj, R.: Measurement of the ultimate shear strength of a metal-ceramic interface. Acta Metall. 37, 1265-1270 (1989) 
2. Barenblatt, G.I.: Scaling. Cambridge Texts in Applied Mathematics. Cambridge University Press, Cambridge (2003)

3. Białas, M., Mróz, Z.: Modelling of progressive interface failure under combined normal compression and shear stress. Int. J. Solids Struct. 42, 4436-4467 (2005)

4. Białas, M., Mróz, Z.: Crack patterns in thin layers under temperature loading. Part II: Cyclic loading. Eng. Fract. Mech. 73, 939-952 (2006)

5. Capozucca, R.: Experimental FRP/SRP-historic masonry delamination. Compos. Struct. 92, 891-903 (2010)

6. Cottone, A., Giambanco, G.: Minimum bond length and size effects in FRP-substrate bonded joints. Eng. Fract. Mech. 76, 1957-1976 (2009)

7. Erdem Alaca, B., Saif, M.T.A., Sehitoglu, H.: On the interface debond at the edge of a thin film on a thick substrate. Acta Mater. 50, 1197-1209 (2002)

8. Gastaldi, F., Kinderlehrer, D.: The partially suported elastic beam. J. Elast. 13, 71-82 (1983)

9. Grande, E., Imbimbo, M., Sacco, E.: Bond behaviour of CFRP laminates glued on clay bricks: experimental and numerical study. Composites B 42, 330-340 (2011)

10. Hsueh, C.H., Yanaka, M.: Multiple film cracking in film/substrate systems with residual stresses and unidirectional loading. J. Mater. Sci. 38, 1809-1817 (2003)

11. Hu, M.S., Evans, A.G.: The cracking and decohesion of thin films on ductile substrates. Acta Metall. 37, 917-925 (1989)

12. Hutchinson, J.W., Suo, Z.: Mixed mode cracking of layered materials. Adv. Appl. Mech. 29, 63-191 (1992)

13. Ivanov, I.V.: Analysis, modelling and optimization of laminated glasses as plane beam. Int. J. Solids Struct. 43, 6887-6907 (2006)

14. Korelc, J.: Multi-language and multi-environment generation of nonlinear finite element codes. Eng. Comput. 18, 312-332 (2002)

15. Korelc, J.: AceFEM. Mathematica Finite Element Enviroment (2009). Available at http://www.fgg. uni-lj.si/Symech/

16. Mathematica Version 6.0, Wolfram Research, Inc., Champaign, IL (2007)

17. Muralidhar, S., Jagota, A., Bennison, S.J., Saigal, S.: Mechanical behaviour in tension of cracked glass bridged by an elastomeric ligament. Acta Mater. 48, 4577-4588 (2000)

18. Palmer, A.C., Rice, J.R.: The growth of slip surfaces in the progressive failure of over-consolidated clay. Proc. R. Soc. Lond. A 332, 527-548 (1973)

19. Schreyer, H.L., Peffer, A.: Fiber pullout based on a one-dimensional model of decohesion. Mech. Mater. 32, 821-836 (2000)

20. Selvadurai, A.P.S., Willner, K.: Surface-stiffened elastic halfspace under the action of a horizontally directed Mindlin force. Int. J. Mech. Sci. 48, 1072-1079 (2006)

21. Selvadurai, A.P.S., Kringos, N., Scarpas, A.: Bond stress development at a surface coating-substrate interface due to the action of a nucleus of thermo-elastic strain. Surf. Coat. Technol. 202, 1704-1711 (2008)

22. Timm, D.H., Guzina, B.B., Voller, V.R.: Prediction of thermal crack spacing. Int. J. Solids Struct. 40, 125-142 (2003)

23. Timoshenko, S.P., Goodier, J.N.: Theory of Elasticity. Engineering Societies Monographs. McGrawHill, New York (1951)

24. Yuan, H., Teng, J.G., Seracino, R., Wu, Z.S., Yao, J.: Full-range behavior of FRP-to-concrete bonded joints. Eng. Struct. 26, 553-565 (2004) 